\title{
Optimal Control Modification for Robust Adaptation of Singularly Perturbed Systems with Slow Actuators
}

\author{
Nhan T. Nguyen* \\ NASA Ames Research Center, Moffett Field, CA 94035 \\ Abraham Ishihara ${ }^{\dagger}$ \\ Carnegie-Mellon University Silicon Valley, Moffett Field, CA 94035 \\ Vahram Stepanyan \\ Mission Critical Technologies, Inc., Moffett Field, CA 94035 \\ Jovan Boskovic ${ }^{\S}$ \\ Scientific Systems Company, Inc., Boston, MA 01801
}

\begin{abstract}
Recently a new optimal control modification has been introduced that can achieve robust adaptation with a large adaptive gain without incurring high-frequency oscillations as with the standard model-reference adaptive control. This modification is based on an optimal control formulation to minimize the $\mathscr{L}_{2}$ norm of the tracking error. The optimal control modification adaptive law results in a stable adaptation in the presence of a large adaptive gain. This study examines the optimal control modification adaptive law in the context of a system with a time scale separation resulting from a fast plant with a slow actuator. A singular perturbation analysis is performed to derive a modification to the adaptive law by transforming the original system into a reduced-order system in slow time. The model matching conditions in the transformed time coordinate results in increase in the feedback gain and modification of the adaptive law.
\end{abstract}

\section{Introduction}

In recent years, adaptive control has been receiving a significant amount of attention. There has been a steady increase in the number of adaptive control applications in a wide range of settings such as aerospace, robotics, process control, etc. ${ }^{1,2}$ The ability to accommodate system uncertainties and to improve fault tolerance of a control system is a major advantage of adaptive control. Nonetheless, adaptive control still faces significant challenges in providing robustness in the presence of unmodeled dynamics and parametric uncertainties. ${ }^{3}$ The ability for an adaptive control algorithm to modify a pre-existing control design is considered a strength and at the same time a weakness. The crash of the X-15 aircraft in $1967^{4}$ serves as a reminder that adaptive control is still viewed with some misgivings despite enormous advances ever since. Over the past several years, various model-reference adaptive control (MRAC) methods have been investigated. ${ }^{5-9}$

In the conventional MRAC framework, the tracking error is generally inversely proportional to the magnitude of the adaptive gain. However, a large adaptive gain can lead to high-frequency oscillations which can excite unmodeled dynamics that could adversely affect the stability of an MRAC law. ${ }^{10}$ Various modifications were developed to increase robustness of MRAC by adding damping to the adaptive law to reduce high-frequency oscillations. Two well-known modifications in adaptive control are the $\sigma$-modification ${ }^{11}$ and $\varepsilon_{1}$ - modification. ${ }^{12}$ These modifications have been used extensively in adaptive control. Recently, a new modification has been introduced that is based on an optimal control formulation to minimize the $\mathscr{L}_{2}$-norm of the tracking error. ${ }^{13}$ The optimality condition results in a damping term in the adaptive law proportional to persistent excitation. The optimal control modification has been shown to be able to achieve fast adaptation with a large adaptive gain without compromising stability robustness while preserving tracking performance. This study extends the development of the optimal control modification adaptive law to the case

\footnotetext{
* Research Scientist, Intelligent Systems Division, Mail Stop 269-1, AIAA Associate Fellow

${ }^{\dagger}$ Research Scientist, Carnegie Mellon University Silicon Valley, NASA Research Park

${ }^{\ddagger}$ Research Scientist, Intelligent Systems Division, Mail Stop 269-3, AIAA Member

${ }^{\S}$ Principal Research Engineer \& Group Leader, Intelligent \& Autonomous Control Systems, AIAA Senior Member
} 
when there exists a time-scale separation between a fast plant and a slow actuator which prevents the plant to follow a reference model even in the presence of adaptive control. A singular perturbation approach is used to separate the time scales of the plant and actuators and then modify the optimal control modification adaptive law to account for the slow actuator in the singularly perturbed system. The singular perturbation approach transform the original system into a reduced-order system in slow time. The model matching condition is applied to the reduced-order system and the reference model in the transformed slow time coordinate that results in changes in the actuator command to accommodate the slow actuator dynamics. The resulting control signal can then track the reference model better than if the actuator command is not modified.

\section{Singularly Perturbed Systems with Slow Actuators}

A direct MRAC problem is posed as follows:

Given a nonlinear plant as

$$
\dot{x}=A x+B\left[u+\Theta^{* \top} \Phi(x)+v(t)\right]
$$

where $x(t):[0, \infty) \rightarrow \mathbb{R}^{n}$ is a state vector, $u(t):[0, \infty) \rightarrow \mathbb{R}^{n}$ is a control vector, $A \in \mathbb{R}^{n \times n}$ and $B \in \mathbb{R}^{n \times n}$ are known matrices such that the pair $(A, B)$ is controllable and furthermore $A$ is Hurwitz, $\Theta^{*} \in \mathbb{R}^{p \times n}$ is an unknown constant weight matrix, and $\Phi(x): \mathbb{R}^{n} \rightarrow \mathbb{R}^{p}$ is a known bounded bounded basis function and is at least piecewise smooth in $x$, and $v(t):[0, \infty) \rightarrow \mathbb{R}^{n}$ is a small unknown bounded disturbance and is differentiable with $\|v(t)\| \leq v_{0} \in \mathbb{R}$ for all $t$.

The controller $u(t)$ is subject to linear dynamics

$$
\dot{u}=\varepsilon \Lambda\left(u-u_{c}\right)
$$

where $u_{c}(t):[0, \infty) \rightarrow \mathbb{R}^{n}$ is an actuator command vector, $\varepsilon$ is a positive constant, and $\Lambda \in \mathbb{R}^{n \times n}$ is a known Hurwitz matrix.

The objective is to design the controller $u(t)$ that enables the plant to follow a reference model

$$
\dot{x}_{m}=A_{m} x_{m}+B_{m} r
$$

where $A_{m} \in \mathbb{R}^{n \times n}$ is Hurwitz and known, $B_{m} \in \mathbb{R}^{n \times n}$ is also known, and $r(t):[0, \infty) \rightarrow \mathbb{R}^{n} \in \mathscr{L}_{\infty}$ is a command vector with $\dot{r} \in \mathscr{L}_{\infty}$.

If the actuator dynamics are sufficiently fast relative to the reference model dynamics, that is, $\varepsilon\|\Lambda\| \gg\left\|A_{m}\right\|$, then the effect of actuator dynamics may be negligible. Then we design a controller $u(t)$ to follow an actuator command as

$$
u_{c}=K_{x} x+K_{r} r-u_{a d}
$$

where $K_{x} \in \mathbb{R}^{n \times n}$ and $K_{r} \in \mathbb{R}^{n \times n}$ are known nominal gain matrices, and $u_{a d} \in \mathbb{R}^{n}$ is a direct adaptive signal.

Defining the tracking error as $e=x_{m}-x$, then the tracking error equation becomes

$$
\dot{e}=\dot{x}_{m}-\dot{x}=A_{m} x_{m}+B_{m} r-A_{m} x+A_{m} x-A x-B K_{x} x-B K_{r} r+B\left[u_{a d}-\Theta^{\top} \Phi(x)-\delta(t)\right]
$$

Assuming the gain matrices $K_{x}$ and $K_{r}$ can be chosen to satisfy the model matching conditions $A+B K_{x}=A_{m}$ and $B K_{r}=B_{m}$, and the adaptive signal $u_{a d}$ chosen as

$$
u_{a d}=\Theta^{\top} \Phi(x)
$$

where $\Theta$ is an estimated weight matrix and $\tilde{\Theta}=\Theta-\Theta^{*}$ is a a weight variation, then

$$
\dot{e}=A_{m} e+B\left[\tilde{\Theta}^{\top} \Phi(x)-v(t)\right]
$$

The unknown weight matrix $\Theta$ can be estimated by a standard $\sigma$-modification model-reference adaptive control as

$$
\dot{\Theta}=-\Gamma\left(\Phi e^{\top} P B+\sigma \Theta\right)
$$

In this study, we are interested in the case of slow actuator dynamics when $\varepsilon \ll 1$ is a small parameter and $\varepsilon\|\Lambda\| \ll\|A\|$. Then $x(t)$ is a fast state and $u(t)$ is a slow state. To decouple the fast and slow states, we perform 
a time-scale separation by applying the singular perturbation method. Toward that end, we consider a slow time transformation

$$
\tau=\varepsilon t
$$

where $\tau$ is a slow time variable.

Then, the plant and actuator models are transformed into a singularly perturbed system as

$$
\begin{gathered}
\varepsilon \frac{d x}{d \tau}=A x+B\left[u+\Theta^{* \top} \Phi(x)+v(t)\right] \\
\frac{d u}{d \tau}=\Lambda\left(u-u_{c}\right)
\end{gathered}
$$

The Tikhonov's theorem can be used to approximate the solution of the singularly perturbed system with the solution of a "reduced-order" system by setting $\varepsilon=0 .{ }^{14}$ Then, $x(u, \varepsilon)$ is on a fast manifold. Thus, the reduced-order system is given by

$$
\begin{gathered}
B^{-1} A x_{0}+u_{0}+\Theta^{* \top} \Phi\left(x_{0}\right)+v\left(\frac{\tau}{\varepsilon}\right)=u_{0}+v\left(\frac{\tau}{\varepsilon}\right)+f\left(x_{0}\right)=0 \\
\frac{d u_{0}}{d \tau}=\Lambda\left(u_{0}-u_{c}\right)
\end{gathered}
$$

where $x_{0}$ and $u_{0}$ are the "outer" solution of the singularly perturbed system.

The term "outer" is in connection with the concept of "boundary layer" or "inner" and "outer" solutions which have the origin in boundary layer theory due to Prandtl. The "boundary layer" solution for the singularly perturbed system is defined by

$$
\begin{gathered}
\dot{x}_{i}=A\left(x_{0}+x_{i}\right)+B\left[\left(u_{0}+u_{i}\right)+\Theta^{* \top} \Phi\left(x_{0}+x_{i}\right)+v\left(\frac{\tau}{\varepsilon}\right)\right]-A x_{0}-B\left[u_{0}+\Theta^{* \top} \Phi\left(x_{0}\right)+v\left(\frac{\tau}{\varepsilon}\right)\right] \\
=A x_{i}+B\left[u_{i}+\Theta^{* \top} \Phi\left(x_{0}+x_{i}\right)-\Theta^{* \top} \Phi\left(x_{0}\right)\right] \\
\dot{u}_{i}=\varepsilon \Lambda\left(u_{0}+u_{i}-u_{c}\right)-\varepsilon \Lambda u_{0}=\varepsilon \Lambda\left(u_{i}-u_{c}\right)
\end{gathered}
$$

The solution of the original system can be obtained by a matched asymptotic expansion method applied to both the inner and outer solutions. ${ }^{15}$ The outer solution is in fact the asymptotic solution of the original system as $t \rightarrow \infty$.

The algebraic solution of Eq. (12) can be expressed in general as

$$
x_{0}=g\left(u_{0}+v\left(\frac{\tau}{\varepsilon}\right)\right)=-f^{-1}\left(u_{0}+v\left(\frac{\tau}{\varepsilon}\right)\right)
$$

assuming $f^{-1}$ exists.

Differentiating Eq. (16) with respect to the slow time variable and then substituting the actuator model into the result yield

$$
\frac{d x_{0}}{d \tau}=\frac{\partial g}{\partial u_{0}} \frac{d u_{0}}{d \tau}+\frac{\partial g}{\partial \delta} \frac{d \delta}{d \tau}=\frac{\partial g}{\partial u_{0}} \Lambda\left(u_{0}-u_{c}\right)+\frac{\partial g}{\partial v} \frac{d v}{d \tau}
$$

From Eq. (12), we have

$$
u_{0}=-B^{-1} A x_{0}-\Theta^{* \top} \Phi\left(x_{0}\right)-v\left(\frac{\tau}{\varepsilon}\right)
$$

Hence, we obtain the following reduced-order plant model constrained by the slow actuator dynamics

$$
\frac{d x_{0}}{d \tau}=\frac{\partial g}{\partial u_{0}} \Lambda\left[-B^{-1} A x_{0}-\Theta^{* \top} \Phi\left(x_{0}\right)-v\left(\frac{\tau}{\varepsilon}\right)-u_{c}\right]+\frac{\partial g}{\partial v} \frac{d v}{d \tau}
$$

From Eq. (12), we also have

$$
\begin{aligned}
& {\left[B^{-1} A+\Theta^{* \top} \frac{d \Phi\left(x_{0}\right)}{d x_{0}}\right] \frac{\partial x_{0}}{\partial u_{0}}=-I} \\
& {\left[B^{-1} A+\Theta^{* \top} \frac{d \Phi\left(x_{0}\right)}{d x_{0}}\right] \frac{\partial x_{0}}{\partial v}=-I}
\end{aligned}
$$

Thus

$$
\frac{\partial x_{0}}{\partial u_{0}}=\frac{\partial g}{\partial u_{0}}=-\left[B^{-1} A+\Theta^{* \top} \frac{d \Phi\left(x_{0}\right)}{d x_{0}}\right]^{-1}
$$




$$
\frac{\partial x_{0}}{\partial v}=\frac{\partial g}{\partial v}=-\left[B^{-1} A+\Theta^{* \top} \frac{d \Phi\left(x_{0}\right)}{d x_{0}}\right]^{-1}
$$

Therefore

$$
\frac{\partial g}{\partial u_{0}}=\frac{\partial g}{\partial v}
$$

Let

$$
A_{s}=B^{-1} A \Lambda B^{-1} A
$$

and if $A_{s}$ is Hurwitz, then the Tikhonov's theorem guarantees that the reduced solution with $\varepsilon>0$ converge to the solution of the original system with $\varepsilon=0$ as $\varepsilon \rightarrow 0 .{ }^{14}$

Note that Eq. (19) satisfies the outer solution of the nonlinear plant model and the actuator dynamics. Because of the slow actuators, the time scale of the response of the plant cannot exceed that of the actuators. Thus, if the reference model is faster than the actuator model, the tracking error cannot be guaranteed to be small even with adaptive control due to the model mismatch. A possible solution is to revise the reference model to match the actuator-constrained plant model, or alternatively to re-design the actuator command to reduce the tracking error.

In this study, we will consider asymptotic solution of the singularly perturbed system. In effect, the inner solution is neglected so that

$$
\begin{aligned}
& x \approx x_{0} \\
& u \approx u_{0}
\end{aligned}
$$

In slow time, the reference model is expressed as

$$
\frac{d x_{m}}{d \tau}=\frac{1}{\varepsilon}\left(A_{m} x_{m}+B_{m} r\right)
$$

Note that since $\partial g / \partial u_{0}$ contains the uncertainty, the control design is quite complicated. In order to simplified the solution, we make an assumption that the uncertainty term is small. That is

$$
\left\|\Theta^{* \top} \frac{d \Phi(x)}{d x}\right\| \ll\left\|B^{-1} A\right\|
$$

Then, using the matrix inversion lemma, we obtain

$$
\begin{aligned}
{\left[B^{-1} A+\Theta^{* \top} \frac{d \Phi(x)}{d x}\right]^{-1}=A^{-1} B-A^{-1} B\left[\left(\Theta^{* \top} \frac{d \Phi(x)}{d x}\right)^{-1}+\left(B^{-1} A\right)^{-1}\right]^{-1} } & A^{-1} B \\
& \approx A^{-1} B-A^{-1} B \Theta^{* \top} \frac{d \Phi(x)}{d x} A^{-1} B
\end{aligned}
$$

Then, we make the following choice for the actuator command signal

$$
u_{c}=K_{x} x+K_{r} r-u_{a d}
$$

where

$$
\begin{gathered}
K_{x}=\Lambda^{-1} B^{-1} A \frac{1}{\varepsilon} A_{m}-B^{-1} A \\
K_{r}=\Lambda^{-1} B^{-1} A \frac{1}{\varepsilon} B_{m}
\end{gathered}
$$

Comparing this controller with the controller when actuator dynamics are fast, the increase in the control gain is estimated as

$$
\frac{\left\|K_{x}\right\|}{\left\|K_{x}^{*}\right\|}=\frac{\|A\|}{\varepsilon\|\Lambda\|}
$$

where $K_{x}^{*}$ is the control gain for fast actuator dynamics.

The closed-loop singularly perturbed system now becomes

$$
\begin{aligned}
\frac{d x}{d \tau}=\left[B^{-1} A+\Theta^{* \top} \frac{d \Phi(x)}{d x}\right]^{-1} B^{-1} A \frac{1}{\varepsilon}\left(A_{m} x+B_{m} r\right)-\left[B^{-1} A+\Theta^{* \top} \frac{d \Phi(x)}{d x}\right]^{-1} \Lambda\left[u_{a d}-\Theta^{* \top} \Phi(x)\right] & +\frac{\partial g}{\partial v}\left[\frac{d v}{d \tau}-\Lambda v\left(\frac{\tau}{\varepsilon}\right)\right]
\end{aligned}
$$


Using the result of the matrix inversion lemma, we get

$$
\begin{array}{r}
\frac{d x}{d \tau}=\left[I-A^{-1} B \Theta^{* \top} \frac{d \Phi(x)}{d x}\right] \frac{1}{\varepsilon}\left(A_{m} x+B_{m} r\right)-\left[B^{-1} A+\Theta^{* \top} \frac{d \Phi(x)}{d x}\right]^{-1} \Lambda\left[u_{a d}-\Theta^{* \top} \Phi(x)\right] \\
+\frac{\partial g}{\partial v}\left[\frac{d v}{d \tau}-\Lambda v\left(\frac{\tau}{\varepsilon}\right)\right]
\end{array}
$$

Then, the adaptive signal $u_{a d}$ can be designed to keep the following expression small by a judicious choice of new basis function $\Phi_{1}(x, r)$ that spans the unknown parameter space $\Theta_{1}^{*}$ such that

$$
-A^{-1} B \Theta^{* \top} \frac{d \Phi(x)}{d x} \frac{1}{\varepsilon}\left(A_{m} x+B_{m} r\right)-\left[B^{-1} A+\Theta^{* \top} \frac{d \Phi(x)}{d x}\right]^{-1} \Lambda\left[u_{a d}-\Theta^{* \top} \Phi(x)\right]=-A^{-1} B \Lambda \tilde{\Theta}_{1}^{\top} \Phi_{1}(x, r)+\varphi(x, r)
$$

where $\varphi(x, r)$ is an approximation error which is to be kept small by a suitable choice of basis functions.

Solving for $u_{a d}$, we get

$$
u_{a d}=-\Lambda^{-1}\left[B^{-1} A+\Theta^{* \top} \frac{d \Phi(x)}{d x}\right] A^{-1} B \Theta^{* \top} \frac{d \Phi(x)}{d x} \frac{1}{\varepsilon}\left(A_{m} x+B_{m} r\right)+\Theta^{* \top} \Phi(x)-A^{-1} B \Lambda \tilde{\Theta}_{1}^{\top} \Phi_{1}(x, r)+\varphi(x, r)
$$

From the assumption in Eq. (29), we can neglect the term $\left(\Theta^{* \top} \frac{d \Phi(x)}{d x}\right)^{2}$. Then, one possible choice for the new basis function could be

$$
\Phi_{1}(x, r)=\left[\begin{array}{lll}
\Phi(x) & \frac{d \Phi(x)}{d x} x & \frac{d \Phi(x)}{d x} r
\end{array}\right]^{\top}
$$

Alternatively, we can use the universal approximation theorem to approximate the uncertainty with a suitable choice of basis functions such as radial basis functions or sigmoidal basis functions ${ }^{16}$

$$
\frac{d x}{d \tau}=\frac{1}{\varepsilon}\left(A_{m} x+B_{m} r\right)-\frac{1}{\varepsilon} B_{1} \tilde{\Theta}_{1}^{\top} \Phi_{1}(x)+\frac{1}{\varepsilon} B_{1} \delta\left(x, \frac{\tau}{\varepsilon}\right)
$$

where $B_{1}=\varepsilon A^{-1} B \Lambda$ and $\delta\left(x, \frac{\tau}{\varepsilon}\right)=\Lambda^{-1} B^{-1} A\left\{\varphi\left(x, r\left(\frac{\tau}{\varepsilon}\right)\right)+\frac{\partial g}{\partial v}\left[\frac{d v}{d \tau}-\Lambda v\left(\frac{\tau}{\varepsilon}\right)\right]\right\}$.

Since $A_{m}$ is Hurwitz and if $\tilde{\Theta}_{1}^{\top}$ is bounded, then the Tikhonov's theorem guarantees that the reduced solution with $\varepsilon>0$ converge to the solution of the original system with $\varepsilon=0$ as $\varepsilon \rightarrow 0$.

\section{Optimal Control Modification Adaptive Law}

The tracking error equation in slow time is obtained as

$$
\frac{d e}{d \tau}=\frac{d x_{m}}{d \tau}-\frac{d x}{d \tau}=\frac{1}{\varepsilon} A_{m} e+\frac{1}{\varepsilon} B_{1}\left[\tilde{\Theta}_{1}^{\top} \Phi_{1}(x, t)-\delta\left(x, \frac{\tau}{\varepsilon}\right)\right]
$$

We are interested in seeking an update law for $\Theta$ that minimizes the following cost function in slow time

$$
J=\lim _{\tau_{f \rightarrow \infty}} \frac{1}{2 \varepsilon} \int_{0}^{\tau_{f}}(e-\Delta)^{\top} Q(e-\Delta) d \tau
$$

subject to Eq. (41) where $\Delta$ represents the unknown theoretical lower bound of the tracking error and $Q=Q^{\top}>0 \in$ $\mathbb{R}^{n \times n}$.

This optimal control problem can be formulated by the Pontryagin's Maximum Principle. Defining a Hamiltonian

$$
H\left(e, \tilde{\Theta}_{1}^{\top} \Phi_{1}\right)=\frac{1}{2 \varepsilon}(e-\Delta)^{\top} Q(e-\Delta)+\frac{1}{\varepsilon} p^{\top}\left(A_{m} e+B_{1} \tilde{\Theta}_{1}^{\top} \Phi_{1}-B_{1} \delta\right)
$$

where $p(\tau):[0, \infty) \rightarrow \mathbb{R}^{n}$ is an adjoint variable, then the necessary condition is obtained as

$$
\frac{d p}{d \tau}=-\nabla H_{e}^{\top}=-\frac{1}{\varepsilon} Q(e-\Delta)-\frac{1}{\varepsilon} A_{m}^{\top} p
$$


with the transversality condition $p\left(\tau_{f}\right)=0$ since $e(0)$ is known. Treatng $\tilde{\Theta}_{1}^{\top} \Phi_{1}$ as a control variable, then the optimality condition is obtained by

$$
\nabla H_{\tilde{\Theta}_{1}^{\top} \Phi_{1}}=\frac{1}{\varepsilon} p^{\top} B_{1}
$$

The adaptive law which provides an optimal control solution can be formulated as a gradient update law as

$$
\frac{d \tilde{\Theta}_{1}}{d \tau}=-\Gamma \nabla H_{\tilde{\Theta}_{1}^{\top}}=-\Gamma \Phi_{1} \nabla H_{\tilde{\Theta}_{1}^{\top} \Phi_{1}}=-\frac{1}{\varepsilon} \Gamma \Phi_{1} p^{\top} B_{1}
$$

where $\Gamma=\Gamma^{\top}>0 \in \mathbb{R}^{n \times n}$ is an adaptive gain matrix.

The solution of $p$ can be obtained using a "sweeping" method ${ }^{17}$ by letting $p=P e+S \Theta_{1}^{\top} \Phi_{1}$, where $P=P^{\top}>0 \in$ $\mathbb{R}^{n \times n}$ and $S \in \mathbb{R}^{n \times n}$. Substituting into the necessary condition yields

$$
\frac{d P}{d \tau} e+\frac{1}{\varepsilon} P\left(A_{m} e+B \tilde{\Theta}_{1}^{\top} \Phi_{1}-B_{1} \delta\right)+\frac{d S}{d \tau} \Theta_{1}^{\top} \Phi_{1}+S \frac{d\left(\Theta_{1}^{\top} \Phi_{1}\right)}{d \tau}=-\frac{1}{\varepsilon} Q(e-\Delta)-\frac{1}{\varepsilon} A_{m}^{\top}\left(P e+S \Theta_{1}^{\top} \Phi_{1}\right)
$$

This results in the following equations obtained by a method of separation of variables

$$
\begin{gathered}
\frac{d P}{d \tau}+\frac{1}{\varepsilon}\left(P A_{m}+A_{m}^{\top} P\right)+\frac{1}{\varepsilon} Q=0 \\
\frac{d S}{d \tau}+\frac{1}{\varepsilon}\left(A_{m}^{\top} S+P B_{1}\right)=0 \\
-\frac{1}{\varepsilon} P B_{1}\left(\Theta_{1}^{* \top} \Phi+\delta\right)+S \frac{d\left(\Theta_{1}^{\top} \Phi\right)}{d \tau}-\frac{1}{\varepsilon} Q \Delta=0
\end{gathered}
$$

For an infinite time-horizon problem when $\tau_{f} \rightarrow \infty$, then $P(\tau) \rightarrow P(0)$ and $S(\tau) \rightarrow S(0)$ for all $t \in[0, \infty)$. So, both $P$ and $S$ can be approximated by their constant solutions where

$$
\begin{gathered}
P A_{m}+A_{m}^{\top} P=-Q \\
S=-A_{m}^{-\top} P B_{1}
\end{gathered}
$$

Without loss of generality, a weighting constant $v>0 \in \mathbb{R}$ is introduced to allow for adjustments of the modification term in the adaptive law. Then, $v=1$ gives an optimal solution. Thus

$$
S=-v A_{m}^{-\top} P B_{1}
$$

Then, the adjoint $p$ is now expressed as

$$
p=P e-v A_{m}^{-\top} P B_{1} \Theta_{1}^{\top} \Phi_{1}
$$

Substituting Eq. (54) into the gradient-based adaptive law yields the adaptive law in slow time

$$
\frac{d \Theta_{1}}{d \tau}=\frac{d \tilde{\Theta}_{1}}{d \tau}=-\frac{1}{\varepsilon} \Gamma\left(\Phi_{1} e^{\top} P B_{1}-v \Phi_{1} \Phi_{1}^{\top} \Theta_{1} B_{1}^{\top} P A_{m}^{-1} B_{1}\right)
$$
law

Converting to regular time by multiplying $\varepsilon$ through Eq. (55) results in the optimal control modification adaptive

$$
\dot{\Theta}_{1}=-\Gamma\left(\Phi_{1} e^{\top} P B_{1}-v \Phi_{1} \Phi_{1}^{\top} \Theta_{1} B_{1}^{\top} P A_{m}^{-1} B_{1}\right)
$$

\section{A. Stability Proof}

We now prove that the optimal control modification adaptive law (56) is stable and results in uniformly bounded tracking error. Toward that end, choose a Lyapunov candidate function

$$
V=e^{\top} P e+\operatorname{trace}\left(\tilde{\Theta}_{1}^{\top} \Gamma^{-1} \tilde{\Theta}_{1}\right)
$$

Evaluating $d V / d \tau$ in slow time yields

$$
\frac{d V}{d \tau}=\frac{1}{\varepsilon} e^{\top}\left(P A_{m}+A_{m}^{\top} P\right) e+\frac{2}{\varepsilon} e^{\top} P B_{1}\left(\tilde{\Theta}_{1}^{\top} \Phi_{1}-\delta\right)-\frac{2}{\varepsilon} \operatorname{trace}\left(\tilde{\Theta}_{1}^{\top} \Phi_{1} e^{\top} P B_{1}-v \tilde{\Theta}_{1}^{\top} \Phi_{1} \Phi_{1}^{\top} \Theta_{1} B_{1}^{\top} P A_{m}^{-1} B_{1}\right)
$$


By the trace property

$$
\operatorname{trace}\left(X^{\top} Y\right)=Y X^{\top}
$$

where $X, Y \in \mathbb{R}^{N}$, then

$$
\begin{aligned}
\frac{d V}{d \tau}=-\frac{1}{\varepsilon} e^{\top} Q e+\frac{2}{\varepsilon} e^{\top} P B_{1} \tilde{\Theta}_{1}^{\top} \Phi-\frac{2}{\varepsilon} e^{\top} P B_{1} \delta-\frac{2}{\varepsilon} e^{\top} P B \tilde{\Theta}_{1}^{\top} \Phi_{1}+\frac{2}{\varepsilon} \nu \Phi_{1}^{\top} \tilde{\Theta}_{1} B_{1}^{\top} P A_{m}^{-1} B \tilde{\Theta}_{1}^{\top} \Phi_{1} & +\frac{2}{\varepsilon} \nu \Phi_{1}^{\top} \Theta_{1}^{*} B_{1}^{\top} P A_{m}^{-1} B \tilde{\Theta}_{1}^{\top} \Phi_{1}
\end{aligned}
$$

$P A_{m}^{-1}$ can be decomposed into a symmetric part $M=\frac{1}{2}\left(P A_{m}^{-1}+A_{m}^{-\top} P\right)=-\frac{1}{2} A_{m}^{-\top} Q A_{m}^{-1}<0$ and an anti-symmetric part $N=\frac{1}{2}\left(P A_{m}^{-1}-A_{m}^{-\top} P\right)$. Then, $P A_{m}^{-1}=M+N$. By the property of a symmetric matrix, if the symmetric part of a matrix is negative definite, then the matrix is also negative definite. Since $M<0$, therefore $P A_{m}^{-1}<0$. Thus

$$
\frac{d V}{d \tau}=-\frac{1}{\varepsilon} e^{\top} Q e-\frac{2}{\varepsilon} e^{\top} P B_{1} \delta+\frac{2}{\varepsilon} \nu \Phi_{1}^{\top} \tilde{\Theta}_{1} B_{1}^{\top}\left(-\frac{1}{2} A_{m}^{-\top} Q A_{m}^{-1}+N\right) B_{1} \tilde{\Theta}_{1}^{\top} \Phi_{1}+\frac{2}{\varepsilon} \nu \Phi_{1}^{\top} \Theta_{1}^{*} B_{1}^{\top} P A_{m}^{-1} B_{1} \tilde{\Theta}_{1}^{\top} \Phi_{1}
$$

Using the property of an anti-symmetric matrix $y^{\top} N y=0, d V / d \tau$ becomes

$$
\frac{d V}{d \tau}=-\frac{1}{\varepsilon} e^{\top} Q e-\frac{2}{\varepsilon} e^{\top} P B_{1} \delta-\frac{1}{\varepsilon} v \Phi_{1}^{\top} \tilde{\Theta}_{1} B_{1}^{\top} A_{m}^{-\top} Q A_{m}^{-1} B_{1} \tilde{\Theta}_{1}^{\top} \Phi_{1}+\frac{2}{\varepsilon} v \Phi_{1}^{\top} \Theta_{1}^{*} B_{1}^{\top} P A_{m}^{-1} B_{1} \tilde{\Theta}_{1}^{\top} \Phi_{1}
$$

$d V / d \tau$ is then bounded by

$$
\begin{aligned}
\frac{d V}{d \tau} \leq-\frac{1}{\varepsilon} \lambda_{\min }(Q)\|e\|^{2}+\frac{2}{\varepsilon} \lambda_{\max }(P)\|e\|\left\|B_{1}\right\| \delta_{0}-\frac{1}{\varepsilon} v \lambda_{\min }\left(B_{1}^{\top} A_{m}^{-\top} Q A_{m}^{-1} B_{1}\right)\left\|\tilde{\Theta}_{1}\right\|^{2}\left\|\Phi_{1}\right\|^{2} & \\
& +\frac{2}{\varepsilon} v \sigma_{\max }\left(B_{1}^{\top} P A_{m}^{-1} B_{1}\right) \Theta_{0}^{*}\|\tilde{\Theta}\|\|\Phi\|^{2}
\end{aligned}
$$

where $\delta_{0}=\sup _{t}\|\delta\|, \Theta_{0}^{*}=\sup _{t}\left\|\Theta_{1}^{*}\right\|$, and $\lambda$ and $\sigma$ denote the eigenvalue and singular value, respectively.

$d V / d \tau$ can also be expressed as

$$
\begin{aligned}
\frac{d V}{d \tau} \leq-\frac{1}{\varepsilon} \lambda_{\min }(Q)\|e\|[\|e\|- & \left.2 \lambda_{\max }(P)\left\|B_{1}\right\| \delta_{0}\right] \\
& -\frac{1}{\varepsilon} v \lambda_{\min }\left(B_{1}^{\top} A_{m}^{-\top} Q A_{m}^{-1} B_{1}\right)\left\|\tilde{\Theta}_{1}\right\|\left\|\Phi_{1}\right\|^{2}\left[\left\|\tilde{\Theta}_{1}\right\|-2 v \sigma_{\max }\left(B_{1}^{\top} P A_{m}^{-1} B_{1}\right) \Theta_{0}^{*}\right]
\end{aligned}
$$

To show that the tracking error $e$ and the weight variation $\tilde{\Theta}$ are bounded, we require $d V / d \tau<0$. Thus, it follows that

$$
\begin{gathered}
\|e\|>\frac{2 \lambda_{\max }(P)\|e\|\left\|B_{1}\right\| \delta_{0}}{\lambda_{\min }(Q)} \\
\left\|\tilde{\Theta}_{1}\right\|>\frac{2 \sigma_{\max }\left(B_{1}^{\top} P A_{m}^{-1} B_{1}\right) \Theta_{0}^{*}}{\lambda_{\min }\left(B_{1}^{\top} A_{m}^{-\top} Q A_{m}^{-1} B_{1}\right)}
\end{gathered}
$$

Hence, there exists a compact set $\mathscr{C}$ where

$$
\mathscr{C}=\left\{(e, \tilde{\Theta}):\|e\| \leq \frac{2 \lambda_{\max }(P)\|e\|\left\|B_{1}\right\| \delta_{0}}{\lambda_{\min }(Q)},\left\|\tilde{\Theta}_{1}\right\| \leq \frac{2 \sigma_{\max }\left(B_{1}^{\top} P A_{m}^{-1} B_{1}\right) \Theta_{0}^{*}}{\lambda_{\min }\left(B_{1}^{\top} A_{m}^{-\top} Q A_{m}^{-1} B_{1}\right)}\right\}
$$

that contains the origin $e=0$ and $\tilde{\Theta}_{1}=0$.

Then $d V / d \tau<0$ outside the compact set $\mathscr{C}$. Thus, any trajectory $e$ and $\tilde{\Theta}_{1}$ starting in $\mathscr{C}$ will remain in $\mathscr{C}$ for all $t .^{18}$ Therefore, the compact set $\mathscr{C}$ is an invariant set. ${ }^{10}$ Also, any trajectory $e$ and $\tilde{\Theta}_{1}$ starting outside the compact set $\mathscr{C}$ will approach the largest invariant set $\mathscr{C}$ as $t \rightarrow \infty .{ }^{18}$ It follows by the LaSalle's Invariance Principle that $e$ and $\tilde{\Theta}_{1}$ are uniformly bounded. Thus, the optimal control modification adaptive law is stable.

From the Lyapunov stability analysis, it is noted that in the absence of persistent excitation, i.e., $\Phi_{1} \Phi_{1}^{\top}=0$ for all $t$ and if $\delta_{0}=0$, then it can be shown by the Barbalat's lemma that $d V / d \tau$ is uniformly continuous and $e \rightarrow 0$ as $t \rightarrow \infty$. ${ }^{19}$ 
In contrast with the $\sigma$-modification adaptive law, even when the persistent excitation is removed, the tracking error does not tend to the origin. ${ }^{20}$

Since $e$ and $\tilde{\Theta}_{1}$ are bounded, the unknown theoretical lower bound of the tracking error $\Delta$ at $t=t_{f} \rightarrow \infty$ is also bounded by

$$
\|\Delta\| \leq \frac{\lambda_{\max }(P)\left\|B_{1}\right\|}{\lambda_{\min }(Q)}\left[\beta+\delta_{0}+\frac{\varepsilon v \eta}{\sigma_{\min }\left(A_{m}\right)}\right]
$$

where $\left\|\Theta^{* \top} \Phi\right\| \leq \beta \in \mathbb{R}$ and $\left\|\frac{d\left(\Theta^{\top} \Phi\right)}{d \tau}\right\| \leq \eta \in \mathbb{R}$ for all $t$.

One unique feature of the optimal control modification is that as the adaptive gain increases and for $v=1$, the system is guaranteed to be bounded. To show this, the optimal control modification adaptive law can be written as

$$
\dot{\Theta}_{1}=-\Gamma \Phi_{1}\left(e^{\top} P-v \Phi_{1}^{\top} \Theta_{1} B_{1}^{\top} P A_{m}^{-1}\right) B_{1}
$$

Then

$$
\dot{\Theta}_{1}^{\top} \Phi_{1}=-B_{1}^{\top}\left(P e-v A_{m}^{-1} P B_{1} \Theta_{1}^{\top} \Phi_{1}\right) \Phi_{1}^{\top} \Gamma \Phi_{1}
$$

Note that $\Phi_{1}^{\top} \Gamma \Phi_{1} \in \mathbb{R}$, so for large $\Phi_{1}^{\top} \Gamma \Phi_{1}$

$$
\lim _{\Phi_{1}^{\top} \Gamma \Phi_{1} \rightarrow \infty} \frac{\dot{\Theta}_{1}^{\top} \Phi_{1}}{\Phi_{1}^{\top} \Gamma \Phi_{1}}=-B_{1}^{\top}\left(P e-v A_{m}^{-\top} P B_{1} \Theta_{1}^{\top} \Phi_{1}\right)=0
$$

the adaptive signal $\Theta_{1}^{\top} \Phi_{1}$ remains bounded and tends to

$$
B_{1} \Theta_{1}^{\top} \Phi_{1} \rightarrow \frac{1}{v} P^{-1} A_{m}^{\top} P e
$$

The tracking error then becomes

$$
\dot{e} \rightarrow\left(A_{m}+\frac{1}{v} P^{-1} A_{m}^{\top} P\right) e-B_{1} \Theta_{1}^{* \top} \Phi_{1}(x, t)-B_{1} \delta(x, t)
$$

which can be written as

$$
\dot{e} \rightarrow-P^{-1}\left[\left(\frac{1+v}{2 v}\right) Q-\left(\frac{1-v}{2 v}\right) S\right] e-B\left(\Theta_{1}^{* \top} \Phi_{1}(x, t)+\delta(x, t)\right)
$$

where $S=A_{m}^{\top} P-P A_{m}$.

In a special case when $\delta(x, t)=0$ and $\Phi_{1}(x)=x$, the system tends to a linear system as $\Phi_{1}^{\top} \Gamma \Phi_{1} \rightarrow \infty$. Furthermore, if $v=1$, then

$$
e(s) \rightarrow-H(s) B \Theta_{1}^{* \top} x(s)
$$

where system transfer function matrix $H(s)=\left(s I+P^{-1} Q\right)^{-1}$ is strictly positive real (SPR) since $H(j \omega)+H^{\top}(-j \omega)>$ 0 as a result of $P^{-1} Q>0$. For a SISO system, the Nyquist plot of a strictly stable transfer function for a SISO system is strictly in the right half plane with a phase shift less than or equal to $\frac{\pi}{2},{ }^{19}$ corresponding to a phase margin of at least $\frac{\pi}{2}$. Thus, one can deduce that the optimal control modification adaptive law is robustly stable with a large adaptive gain.

\section{B. Example}

Consider the following simple scalar system

$$
\dot{x}=a x+b u+\theta^{*} x+v(t)
$$

with actuator dynamics

$$
\dot{u}=\varepsilon \lambda\left(u-u_{c}\right)
$$

where $a<0, \lambda<0, \varepsilon>0,|\varepsilon \lambda|<|a|$, and $v(t)$ is a disturbance signal.

The reference model is

$$
\dot{x}_{m}=a_{m} x_{m}+b_{m} r
$$


where $a_{m}<0$.

The actuator command is designed as

$$
u_{c}=\frac{a}{b}\left(\frac{a_{m}}{\varepsilon \lambda}-1\right) x+\frac{a}{b} \frac{b_{m}}{\varepsilon \lambda} r-\Theta^{\top} \Phi(x, r)
$$

where $\Phi(x, r)=\left[\begin{array}{ll}x & r\end{array}\right]^{\top}$.

Note that if actuator dynamics are fast then the actuator command is

$$
u_{c}=\frac{a}{b}\left(\frac{a_{m}}{a}-1\right) x+\frac{b_{m}}{b} r-\theta x
$$

The optimal control modification update law for slow actuator system is

$$
\dot{\Theta}=-\Gamma\left(\Phi e p b_{1}-v \Phi \Phi^{\top} \Theta_{1} b_{1}^{2} \frac{p}{a_{m}}\right)=-\varepsilon \Gamma\left(\Phi e p \frac{b \lambda}{a}-\varepsilon v \Phi \Phi^{\top} \Theta_{1} \frac{b^{2} \lambda^{2}}{a^{2}} \frac{p}{a_{m}}\right)
$$

where $b_{1}=\frac{b \varepsilon \lambda}{a}$ and $p=-\frac{1}{a_{m}}$, and for fast actuator system is

$$
\dot{\theta}=-\Gamma\left(x e p b-v x^{2} \theta b^{2} \frac{p}{a_{m}}\right)
$$

If $a$ and $\lambda$ are nominally in the same order of magnitude, then we note that for the slow actuator system, the effective adaptive gain is also reduced by $\varepsilon$ for a similar performance as that for the fast actuator.

For the numerical example, $a=-1, b=1, \theta^{*}=0.1, \lambda=-1, \varepsilon=0.1, a_{m}=-5, b_{m}=1, r(t)=\sin t, v(t)=$ $0.05 \sin 10 t$. The responses due to the standard MRAC adaptive law and optimal control modification adaptive law with the singular perturbation approach are plotted in Fig. 1. The response for the standard MRAC exhibits more initial transient than that for optimal control modification using the same adaptive gain.
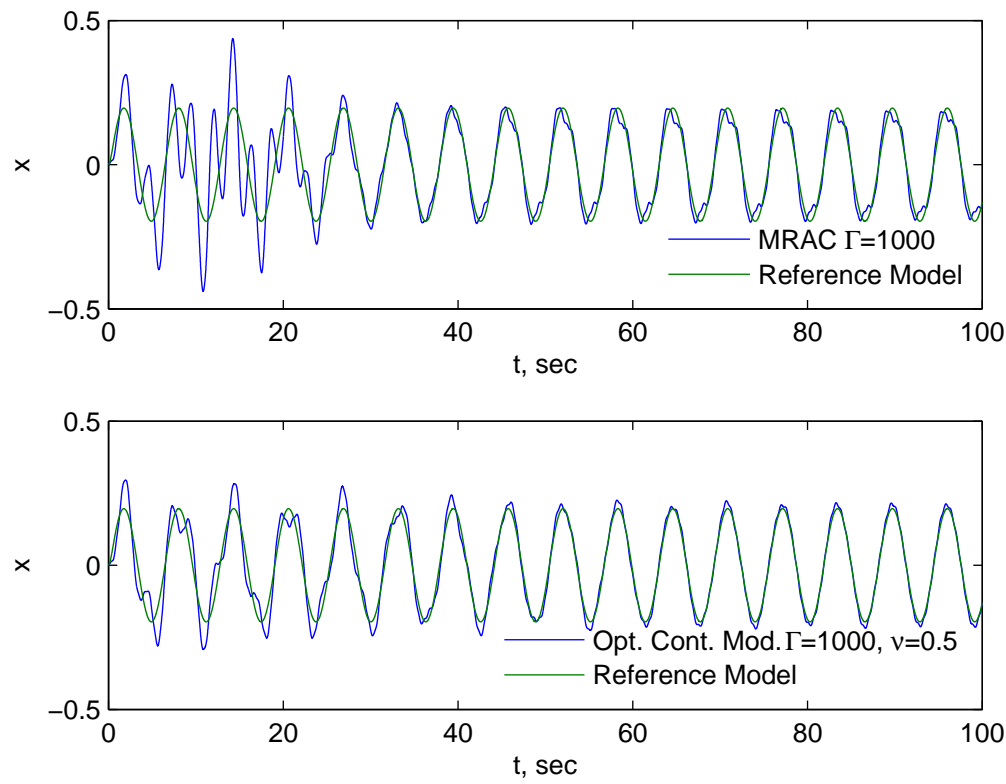

Fig. 1 - Responses due to MRAC and Optimal Control Modification

Figure 2 is a plot of the control input and actuator command with the singular perturbation approach. As can be seen, the actuator command signal is quite large relative to the control input. This is due to the fact that the actuator dynamics are slow so a large actuator command does not translate into the same amount of control input for a finite time. The effectiveness of the optimal control modification is demonstrated by reducing the amplitude of oscillation in the control input significantly over that due to the standard MRAC. 

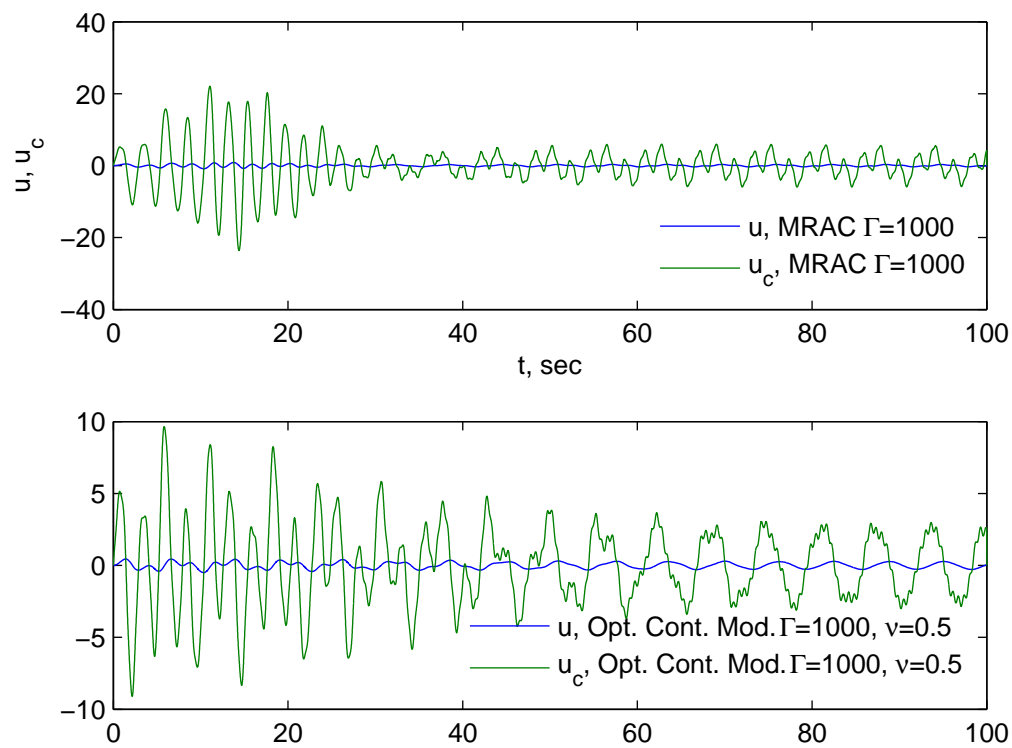

Fig. 2 - Control and Actuator Command due to MRAC and Optimal Control Modification

Figure shows the responses due to the unmodified actuator command for fast actuator dynamics. As can be seen, the control input is insufficient to allow the plant to follow the reference model even with adaptive control.
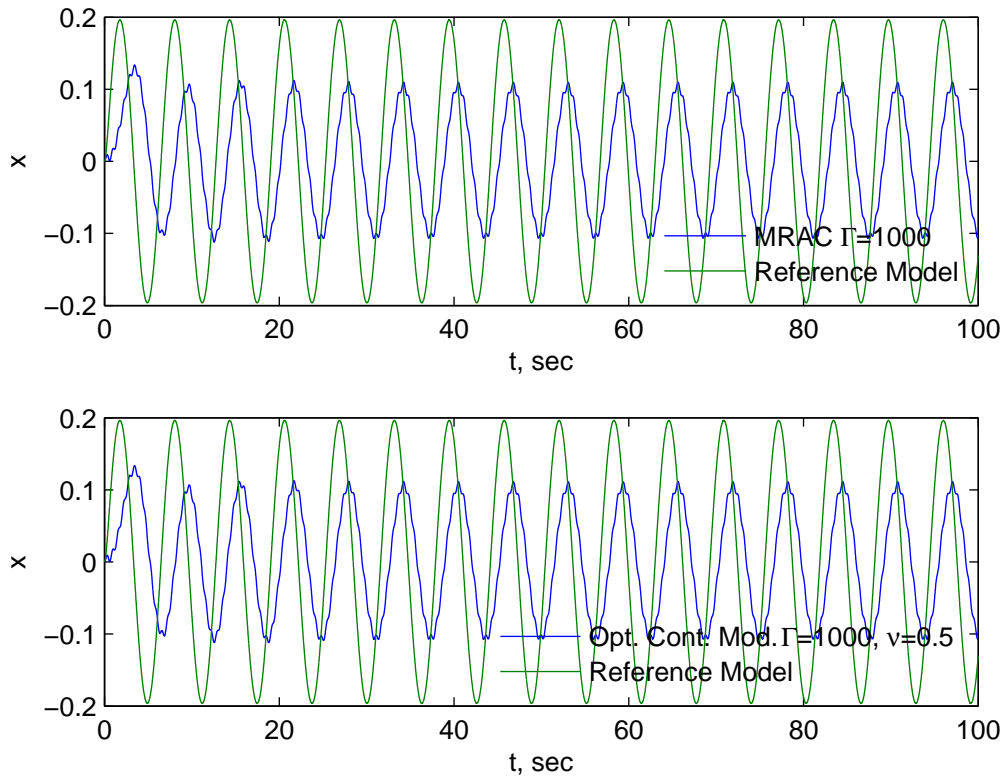

Fig. 3 - Responses due to Un-modified Actuator Command for Slow Dynamics

\section{Flight Control Application}

Consider the following inner loop adaptive flight control architecture as shown in Fig. 1. The control architecture comprises: 1) a reference model that translates rate commands into desired acceleration commands, 2) a proportionalintegral (PI) feedback control for rate stabilization and tracking, 3) a dynamic inversion controller that computes actuator commands using desired acceleration commands, 4) an adaptive controller with the conventional MRAC 
law and with or without the optimal control modification adaptive law, and 5) a parameter estimator for actuator dynamics as relative to its nominal dynamics via the parameter $\varepsilon$. The adaptive controller is designed to increase performance of the nominal dynamic inversion controller under adverse flight conditions such as upsets and damage. Under nominal fast actuator dynamics, both the dynamic inversion control and the adaptive law are computed without any differentiation between actuator commands and control inputs. As actuator dynamics degrade based on the value of $\varepsilon$, both the dynamic inversion controller and the adaptive controller are modified accordingly to increase the actuator command signals.

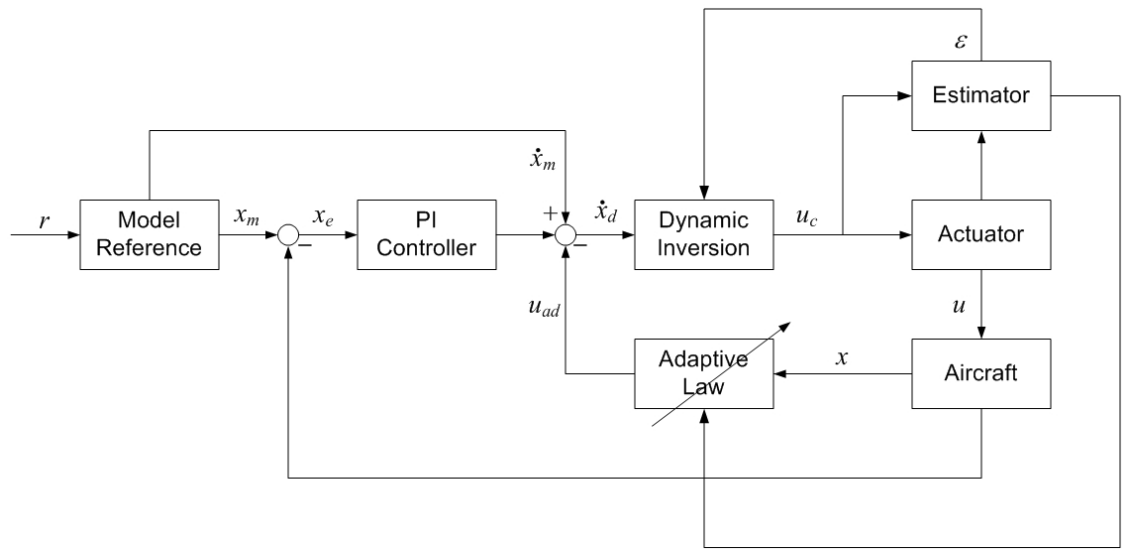

Fig. 4 - Adaptive Flight Control Architecture

The linearized equations of motion are expressed as

$$
\begin{aligned}
& \dot{x}=A_{11} x+A_{12} z+B_{1} u+f_{1}(x, z) \\
& \dot{z}=A_{21} x+A_{22} z+B_{2} u+f_{2}(x, z)
\end{aligned}
$$

where $A_{i j}$ and $B_{i}, i=1,2$ are known, $x=\left[\begin{array}{lll}p & q & r\end{array}\right]^{\top}$ is a vector of roll, pitch, and yaw rates; $z=\left[\begin{array}{cccccc}\Delta \phi & \Delta \alpha & \Delta \beta & \Delta V & \Delta h & \Delta \theta\end{array}\right]^{\top}$ is a vector of perturbation in the bank angle $\Delta \phi$, angle of attack $\Delta \alpha$, sideslip angle $\Delta \beta$, airspeed $\Delta V$, altitude $\Delta h$, and pitch angle $\Delta \theta ; u=\left[\begin{array}{ccc}\Delta \delta_{a} & \Delta \delta_{e} & \Delta \delta_{r}\end{array}\right]^{\top}$ is a vector of additional aileron, elevator, and rudder deflections; and $f_{i}(x, z), i=1,2$ are parametric uncertainties which can be expressed as

$$
f_{i}(x, z)=\Theta_{i}^{* \top} \Phi(x, z)+\delta_{i}(x, z)
$$

where $\delta(x, z)$ is an approximation error which is assumed to be small by a suitable choice of basis functions, $\Phi=$ $\left[\begin{array}{llll}C_{1} & C_{2} & C_{3} & C_{4}\end{array}\right]^{\top}$ is a basis function for a sigma-pi neural network with $C_{i}, i=1, \ldots, 4$, as inputs consisting of control commands, sensor feedback, and bias terms; defined as follows:

$$
\begin{aligned}
& C_{1}=\frac{1}{2} \rho(h) V^{2}\left[\begin{array}{lll}
x^{\top} & \alpha x^{\top} & \beta x^{\top}
\end{array}\right] \\
& C_{2}=\frac{1}{2} \rho(h) V^{2}\left[\begin{array}{llllllll}
1 & \phi & \theta & \alpha & \beta & \alpha^{2} & \beta^{2} & \alpha \beta
\end{array}\right] \\
& C_{3}=\frac{1}{2} \rho(h) V^{2}\left[\begin{array}{lll}
u(x, z)^{\top} & \alpha u(x, z)^{\top} & \beta u(x, z)^{\top}
\end{array}\right] \\
& C_{4}=\frac{1}{2} \rho(h) V^{2}\left[\begin{array}{lll}
p x^{\top} & q x^{\top} & r x^{\top}
\end{array}\right]
\end{aligned}
$$

where $\phi=\bar{\phi}+\Delta \phi, \alpha=\bar{\alpha}+\Delta \alpha, \beta=\bar{\beta}+\Delta \beta, V=\bar{V}+\Delta V, h=\bar{h}+\Delta h$, and $\theta=\bar{\theta}+\Delta \theta$; and the overbar symbol denotes a trim state. 
These inputs are designed to model the parametric uncertainty that exists in the damaged aircraft plant dynamics. For example, the aerodynamic force in the $x$-axis for an aircraft is given by

$$
\begin{aligned}
F_{x}=T+\frac{1}{2} \bar{q} S\left(C_{L_{0}}+C_{L_{\alpha}} \alpha+C_{L_{\beta}} \beta+C_{L_{p}} \frac{p b}{2 V}+C_{L_{q}} \frac{q \bar{c}}{2 V}+C_{L_{r}} \frac{r b}{2 V}+C_{L_{\delta_{a}}} \delta_{a}+C_{L_{\delta_{e}}} \delta_{e}+C_{L_{\delta_{r}}} \delta_{r}\right) \alpha \\
-\frac{1}{2} \bar{q} S\left(C_{D_{0}}+C_{D_{\alpha}} \alpha+C_{D_{p}} \frac{p b}{2 V}+C_{D_{q}} \frac{q \bar{c}}{2 V}+C_{D_{r}} \frac{r b}{2 V}+C_{D_{\delta_{a}}} \delta_{a}+C_{D_{\delta_{e}}} \delta_{e}+C_{D_{\delta_{r}}} \delta_{r}\right)
\end{aligned}
$$

Thus, $C_{1}, C_{2}$, and $C_{3}$ are designed to model the product terms of $x, z$, and $u$ in the aerodynamic forces and moments equations; and $C_{4}$ models the gyroscopic cross-coupling terms of $x$ in the moment equations.

The inner loop rate feedback control is designed to improve aircraft rate response characteristics such as the short period mode and the dutch roll mode. A second-order reference model is specified to provide desired handling qualities with good damping and natural frequency characteristics as follows:

$$
\begin{gathered}
\left(s^{2}+2 \zeta_{p} \omega_{p} s+\omega_{p}^{2}\right) \phi_{m}=g_{p} \delta_{l a t} \\
\left(s^{2}+2 \zeta_{q} \omega_{q} s+\omega_{q}^{2}\right) \theta_{m}=g_{q} \delta_{l o n} \\
\left(s^{2}+2 \zeta_{r} \omega_{r} s+\omega_{r}^{2}\right) \beta_{m}=-g_{r} \delta_{r u d}
\end{gathered}
$$

where $\phi_{m}, \theta_{m}$, and $\psi_{m}$ are reference bank, pitch, and sideslip angles; $\omega_{p}, \omega_{q}$, and $\omega_{r}$ are the natural frequencies for desired handling qualities in the roll, pitch, and yaw axes; $\zeta_{p}, \zeta_{q}$, and $\zeta_{r}$ are the desired damping ratios; $\delta_{l a t}, \delta_{l o n}$, and $\delta_{r u d}$ are the lateral stick input, longitudinal stick input, and rudder pedal input; and $g_{p}, g_{q}$, and $g_{r}$ are input gains.

Let $p_{m}=\dot{\phi}_{m}, q_{m}=\dot{\theta}_{m}$, and $r_{m}=-\dot{\beta}_{m}$ be the reference roll, pitch, and yaw rates. Then the reference model can be described as

$$
\dot{x}_{m}=-K_{p} x_{m}-K_{i} \int_{0}^{t} x_{m} d \tau+G r
$$

where $x_{m}=\left[\begin{array}{lll}p_{m} & q_{m} & r_{m}\end{array}\right]^{\top}, K_{p}=\operatorname{diag}\left(2 \zeta_{p} \omega_{p}, 2 \zeta_{q} \omega_{q}, 2 \zeta_{r} \omega_{r}\right), K_{i}=\operatorname{diag}\left(\omega_{p}^{2}, \omega_{q}^{2}, \omega_{r}^{2}\right), G=\operatorname{diag}\left(g_{p}, g_{q}, g_{r}\right)$, and $r=\left[\begin{array}{lll}\delta_{\text {lat }} & \delta_{\text {lon }} & \delta_{\text {rud }}\end{array}\right]^{\top}$.

Suppose, the elevator actuator is a slow actuator where

$$
\dot{\delta}_{e}=\varepsilon \lambda_{e}\left(\delta_{e}-\delta_{e c}\right)
$$

where $\lambda_{e}<0$ is the original elevator actuator rate, $\delta_{e c}$ is the elevator deflection command, and $\varepsilon>0$ is unknown but can be estimated by a suitable parameter estimation technique such as the recursive least-squares method if the control signal $u(t)$ and its derivative $\dot{u}(t)$ is available by estimation, in which case $u(t)$ is replaced by $\hat{u}(t)$, or by direct measurement. A recursive least-squares algorithm can be used to estimate $\varepsilon$ as follows:

$$
\begin{gathered}
\dot{\varepsilon}=R \Delta u\left(\dot{\hat{u}}^{\top}-\Delta u^{\top} \Lambda^{\top} \varepsilon\right) \\
\dot{R}=-R \Delta u \Delta u^{\top} R
\end{gathered}
$$

where $\Delta u=\hat{u}-u_{c}$.

Assuming the pair $\left(A_{11}, B_{1}\right)$ is controllable and $z$ is stabilizable, the reduced-order equation for the pitch rate is

$$
0=E_{2} B_{1}^{-1} A_{11} x+E_{2} B_{1}^{-1} A_{12} z+E_{2} u+E_{2} B_{1}^{-1} f_{1}
$$

where $E_{1}=\left[\begin{array}{lll}1 & 0 & 0\end{array}\right], E_{2}=\left[\begin{array}{lll}0 & 1 & 0\end{array}\right]$, and $E_{3}=\left[\begin{array}{ccc}0 & 0 & 1\end{array}\right]$ are basis vectors.

Note that $E_{2} u=\delta_{e}$ and $E_{2} u_{c}=\delta_{e c}$, then differentiating the reduced-order equation gives

$$
E_{2} B_{1}^{-1} A_{11} \dot{x}+E_{2} B_{1}^{-1} A_{12} \dot{z}=\varepsilon \lambda_{e}\left(E_{2} B_{1}^{-1} A_{11} x+E_{2} B_{1}^{-1} A_{12} z+E_{2} B_{1}^{-1} f_{1}+E_{2} u_{c}\right)
$$

Setting $\dot{x}=-\left(K_{p}+\frac{K_{i}}{s}\right) x+G r$, then the actuator commands can be computed as follows

$$
u_{c}=\left[\begin{array}{c}
E_{1} B_{1}^{-1} \\
\frac{1}{\varepsilon \lambda_{e}} E_{2} B_{1}^{-1} A_{11} \\
E_{3} B_{1}^{-1}
\end{array}\right]_{3 \times 3}\left[-\left(K_{p}+\frac{K_{i}}{s}\right) x+G r\right]+\left[\begin{array}{c}
0 \\
\frac{1}{\varepsilon \lambda_{e}} E_{2} B_{1}^{-1} A_{12} \\
0
\end{array}\right]_{3 \times 3} \dot{z}-B^{-1}\left(A_{11} x+A_{12} z+\Theta_{1}^{\top} \Phi\right)
$$


where $u_{c}=\left[\begin{array}{lll}\Delta \delta_{a c} & \Delta \delta_{e c} & \Delta \delta_{r c}\end{array}\right]^{\top}$.

The closed-loop angular rate dynamics are then given by

$$
\dot{x}=-\left(K_{p}+\frac{K_{i}}{s}\right) x+G r-\left[\begin{array}{c}
E_{1} \\
\varepsilon \lambda_{e} E_{2} A_{11}^{-1} \\
E_{3}
\end{array}\right]_{3 \times 3}\left(\tilde{\Theta}_{1}^{\top} \Phi-\delta_{1}\right)
$$

Let $e=\left[\begin{array}{ll}\int_{0}^{t}\left(x_{m}-x\right) d \tau & x_{m}-x\end{array}\right]^{\top}$ be the tracking error, then the tracking error equation is given by

$$
\dot{e}=A_{m} e+B\left(\tilde{\Theta}_{1}^{\top} \Phi-\delta_{1}\right)
$$

where

$$
\begin{gathered}
A_{m}=\left[\begin{array}{cc}
0 & I \\
-K_{i} & -K_{p}
\end{array}\right] \\
B=\left[\begin{array}{c}
0 \\
E
\end{array}\right] \\
E=\left[\begin{array}{c}
E_{1} \\
\varepsilon \lambda_{e} E_{2} A_{11}^{-1} \\
E_{3}
\end{array}\right]_{3 \times 3}
\end{gathered}
$$

Let $Q=2 I$, then the solution of Eq. (51) yields

$$
P=\left[\begin{array}{cc}
K_{i}^{-1} K_{p}+K_{p}^{-1}\left(K_{i}+I\right) & K_{i}^{-1} \\
K_{i}^{-1} & K_{p}^{-1}\left(I+K_{i}^{-1}\right)
\end{array}\right]>0
$$

$A_{m}^{-1}$ is computed to be

$$
A_{m}^{-1}=\left[\begin{array}{cc}
-K_{i}^{-1} K_{p} & -K_{i}^{-1} \\
I & 0
\end{array}\right]
$$

Evaluating the term $B^{\top} P A_{m}^{-1} B$ yields

$$
B^{\top} P A_{m}^{-1} B=-E^{\top} K_{i}^{-2} E<0
$$

Applying the adaptive optimal control modification (56), the weight update law is then given by

$$
\dot{\Theta}_{1}=-\Gamma \Phi\left(e^{\top} P B+v \Phi^{\top} \Theta_{1} E^{\top} K_{i}^{-2} E\right)
$$

\section{A. Simulation Results}

A simulation study was conducted using a generic transport model (GTM) which represents a notational twin-engine transport aircraft as shown in Fig. 5. ${ }^{21}$ An aerodynamic model of the damaged aircraft is created using a vortex lattice method to estimate aerodynamic coefficients, and stability and control derivatives. For the simulation, a damage configuration is modeled corresponding to a $28 \%$ loss of the left wing. The damage causes an estimated C.G. shift mostly along the pitch axis with $\Delta y=0.0388 \bar{c}$ and an estimated mass loss of $1.2 \%$. The principal moment of inertia about the roll axis is reduced by $12 \%$, while changes in the inertia values in the other two axes are not as significant. Since the damaged aircraft is asymmetric, the inertia tensor has all six non-zero elements. This means that all the three roll, pitch, and yaw axes are coupled together throughout the flight regime. In addition, the elevator actuator is simulated as an impaired flight control actuator with a much smaller bandwidth than that for a healthy actuator. 


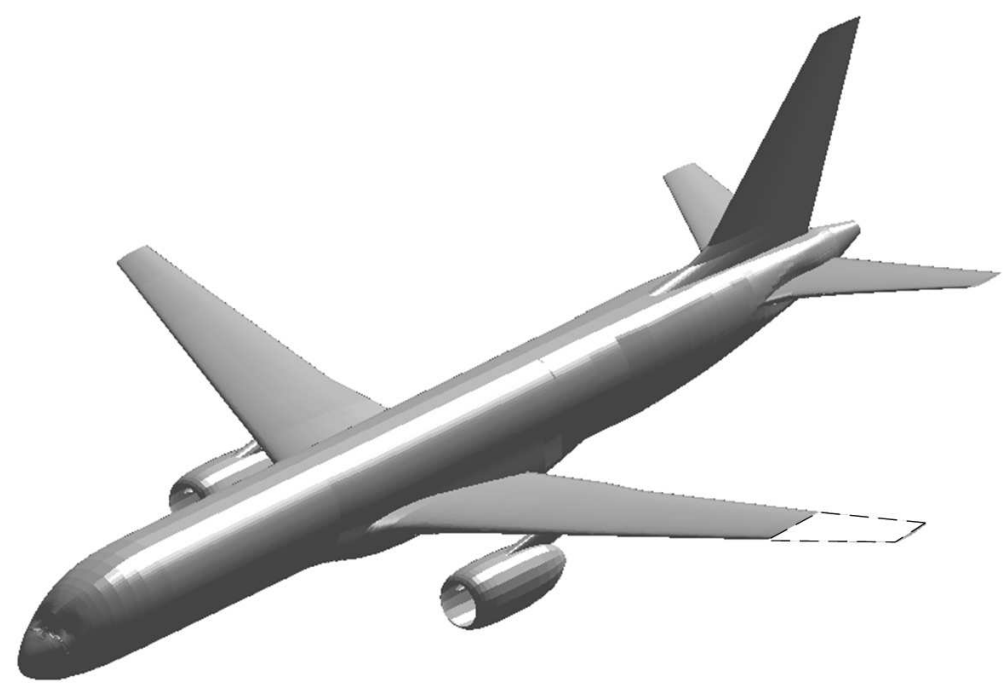

Fig. 5 - Left Wing Damaged Generic Transport Model

A level flight condition of Mach 0.6 at $15,000 \mathrm{ft}$ is selected. Upon damage, the aircraft is re-trimmed with $T=$ $13,951 \mathrm{lb}, \bar{\alpha}=5.86^{\circ}, \bar{\phi}=-3.16^{\circ}, \bar{\delta}_{a}=27.32^{\circ}, \bar{\delta}_{e}=-0.53^{\circ}, \bar{\delta}_{r}=-1.26^{\circ}$. The remaining right aileron is the only roll control effector available. In practice, some aircraft can control a roll motion with spoilers, which are not modeled in this study. The reference model is specified by $\omega_{p}=2.0 \mathrm{rad} / \mathrm{sec}, \omega_{q}=1.5 \mathrm{rad} / \mathrm{sec}, \omega_{r}=1.0 \mathrm{rad} / \mathrm{sec}$, and $\zeta_{p}=\zeta_{q}=\zeta_{r}=1 / \sqrt{2}$. The simulations also include a random signal to represent sensor noise.

The actuator dynamics are modeled with $\lambda_{a}=\lambda_{e}=\lambda_{r}=50 / \mathrm{sec}$ with position limits of $\pm 35^{\circ}$ for the aileron and elevator and $\pm 10^{\circ}$ for the rudder. The $\varepsilon$ parameter for the elevator is set to 0.01 .

The pilot pitch rate command is simulated with a series of ramp input longitudinal stick command doublets, corresponding to the reference pitch angle $\pm 3.81^{\circ}$ from trim. At $t=10 \mathrm{sec}$, a wing damage and elevator actuator degradation occur. The tracking performance of the baseline flight controller, which is a proportional-integral feedback type with no adaptation, is compared against the optimal control modification adaptive law with and without the singular perturbation approach for slow actuators. Both the adaptive laws are implemented as an augmentation to the baseline controller. An adaptive gain of $\Gamma=60$ and a weighting factor $v=0.2$ are selected.

The aircraft angular rate responses are shown in Figs. 6 to 9. Figure 6 illustrates the pitch rate responses. With no adaptation, the baseline controller cannot follow the reference pitch rate very well. The pitch rate response clearly lags the reference model significantly due to the degraded elevator actuator. The optimal control modification with and without the singular perturbation approach significantly improve the tracking and reduces the lag between the response and the reference model. The pitch rate is worse without than with the singular perturbation approach, as large initial transients occur at failure. So, the singular perturbation approach demonstrates an improved response due to the slow elevator actuator.

Since the damage occurs to one of the wings, the roll axis is most affected. With no adaptation, there is a significant roll rate as high as $20^{\circ} / \mathrm{sec}$ as shown in Fig. 7. There is a steady-state oscillation of $\pm 15^{\circ} / \mathrm{sec}$ in the roll rate. Both the optimal control modification adaptive laws reduce the oscillation to within $\pm 2 \%$ sec. However, there is a large initial transient of about $20^{\circ} / \mathrm{sec}$ due to the sudden wing loss.

Figure 8 is the yaw rate response of the damaged aircraft. The optimal control modification with the singular perturbation approach slightly improves the yaw rate response than that without. Both adaptive laws result in a much lower yaw rate than the baseline controller.

Figure 9 is the plot of the tracking error $\mathscr{L}_{2}$ norms for the three axes in combination. Not surprisingly, without the adaptation, the baseline controller suffers a large tracking error. 

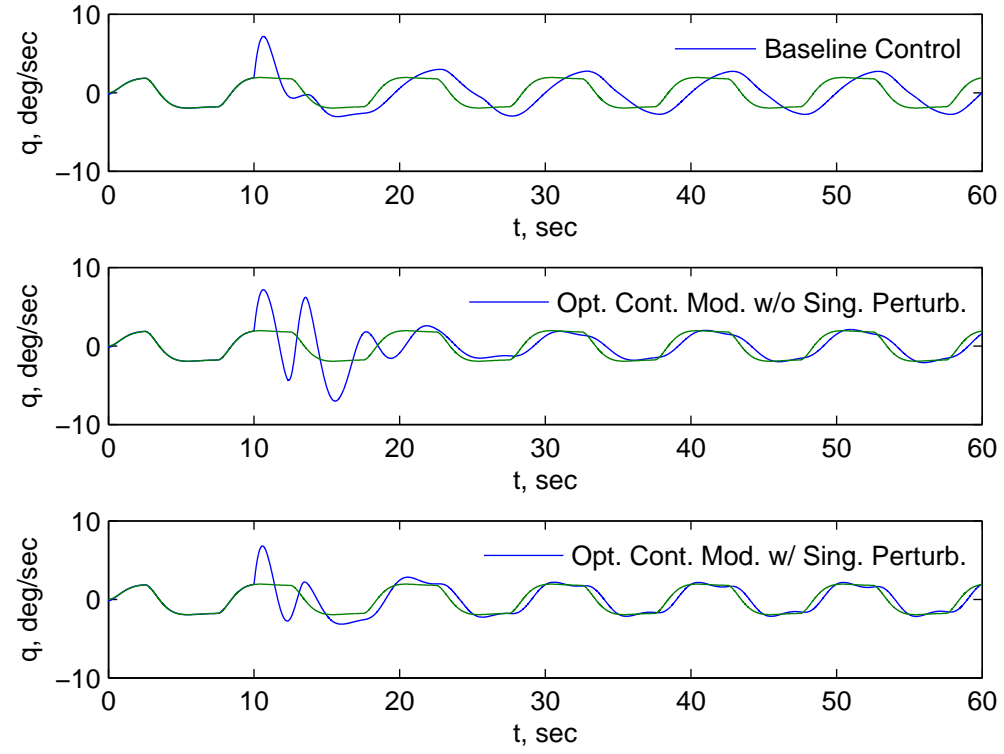

Fig. 6 - Pitch Rate
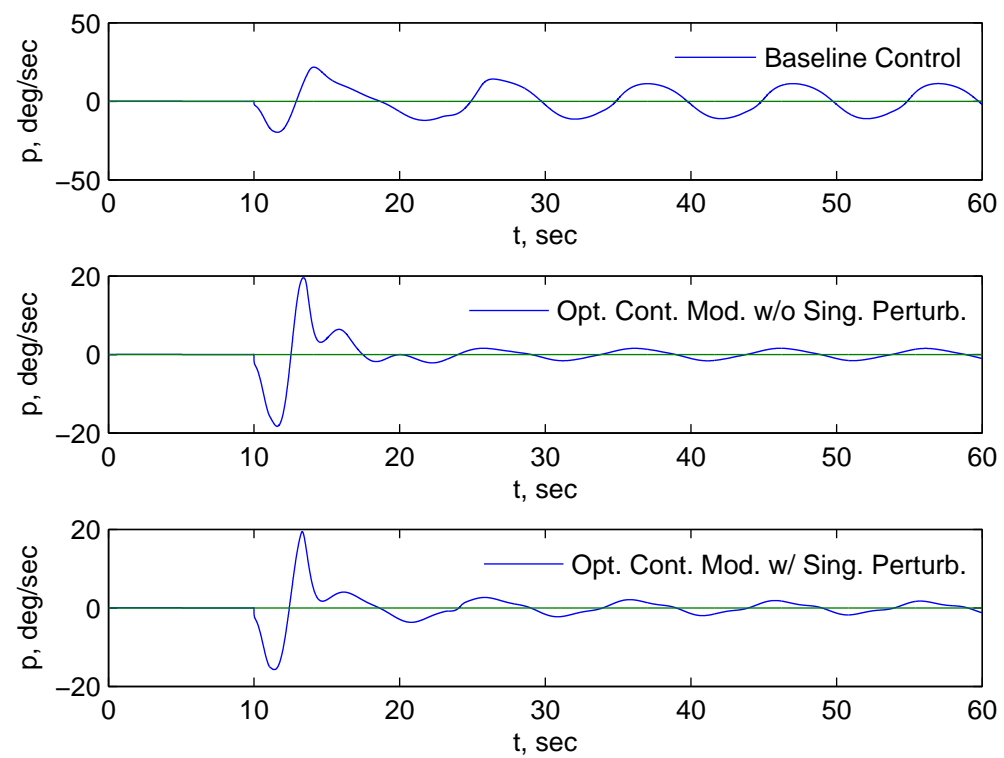

Fig. 7 - Roll Rate 

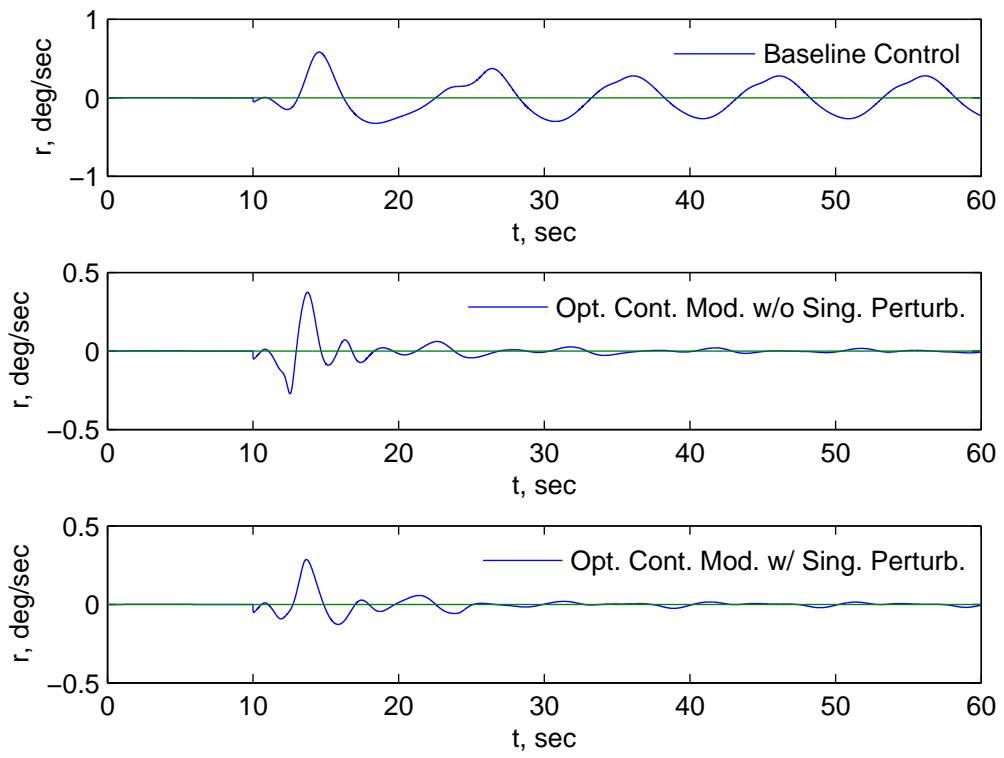

Fig. 8 - Yaw Rate
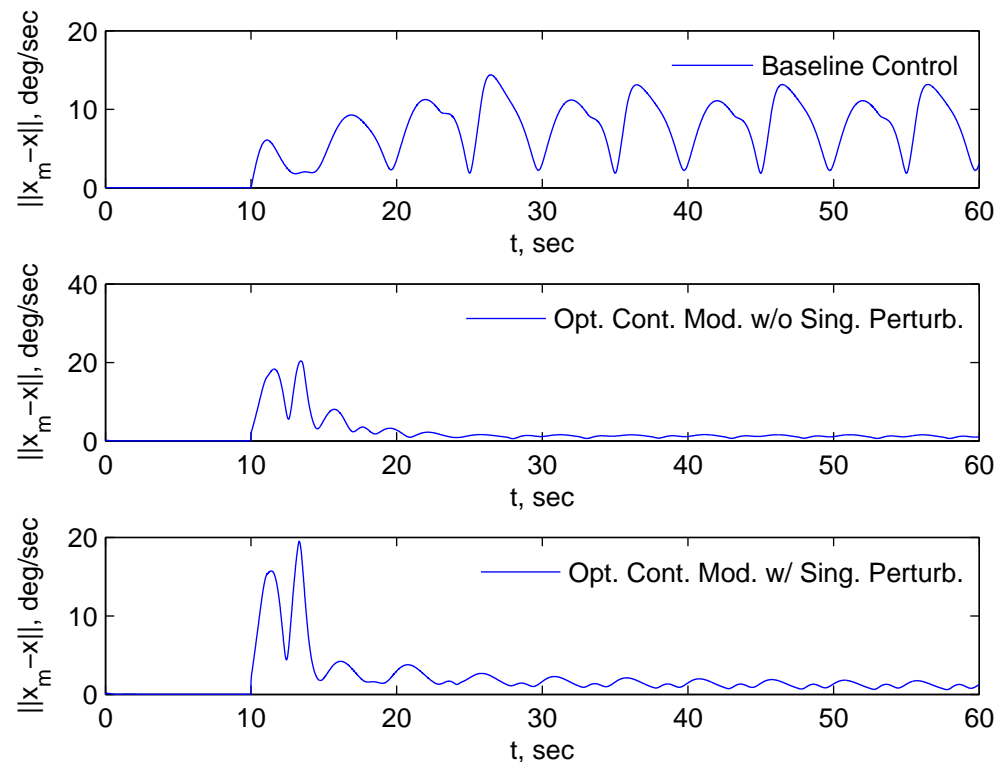

Fig. 9 - Tracking Error Norm

The control surface deflections are plotted in Figs. 10 to 12. The aileron actuator is a fast acting actuator, so in all cases, the aileron deflection tracks the command as shown in Fig. 10. With both the optimal control modification adaptive laws, there is a large aileron command at the instance of failure that causes the aileron to saturate. The spike in the aileron command is larger without than with the singular perturbation approach. 

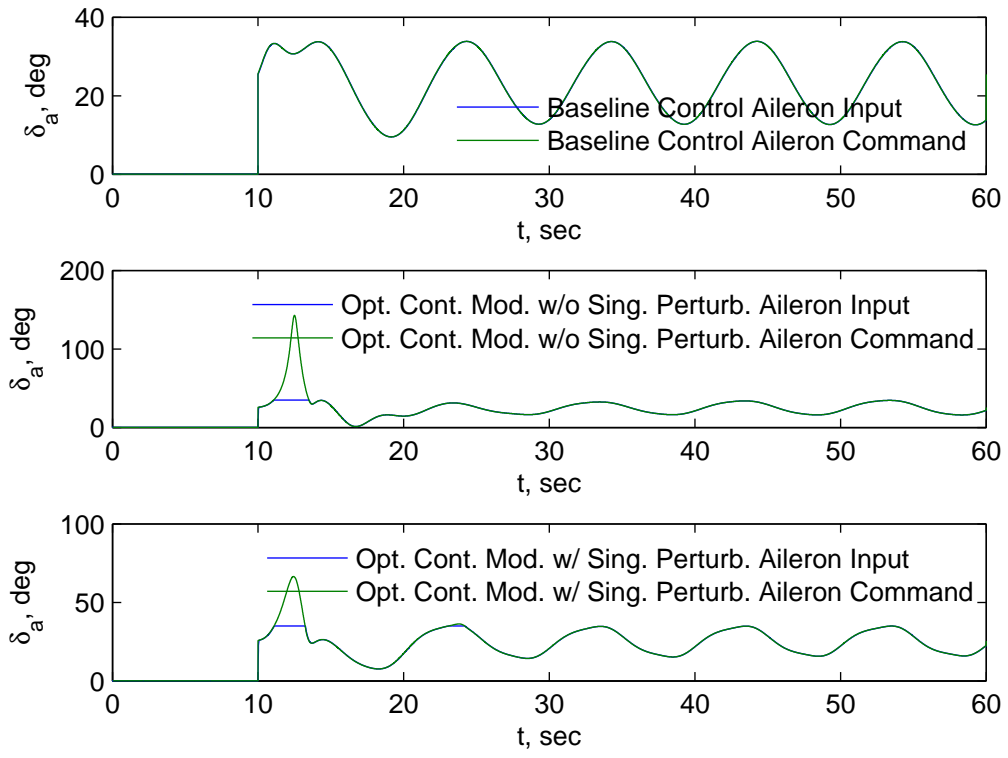

Fig. 10 - Aileron Deflection

Figure 11 is a plot of the elevator deflection. Since the aileron actuator is degraded, it is clear that the elevator command cannot be tracked well by the elevator actuator. The optimal control modification adaptive law without the singular perturbation approach produces a large initial spike in the elevator command of about $-25^{\circ}$ while the initial transient in the command is reasonable with the singular perturbation approach.
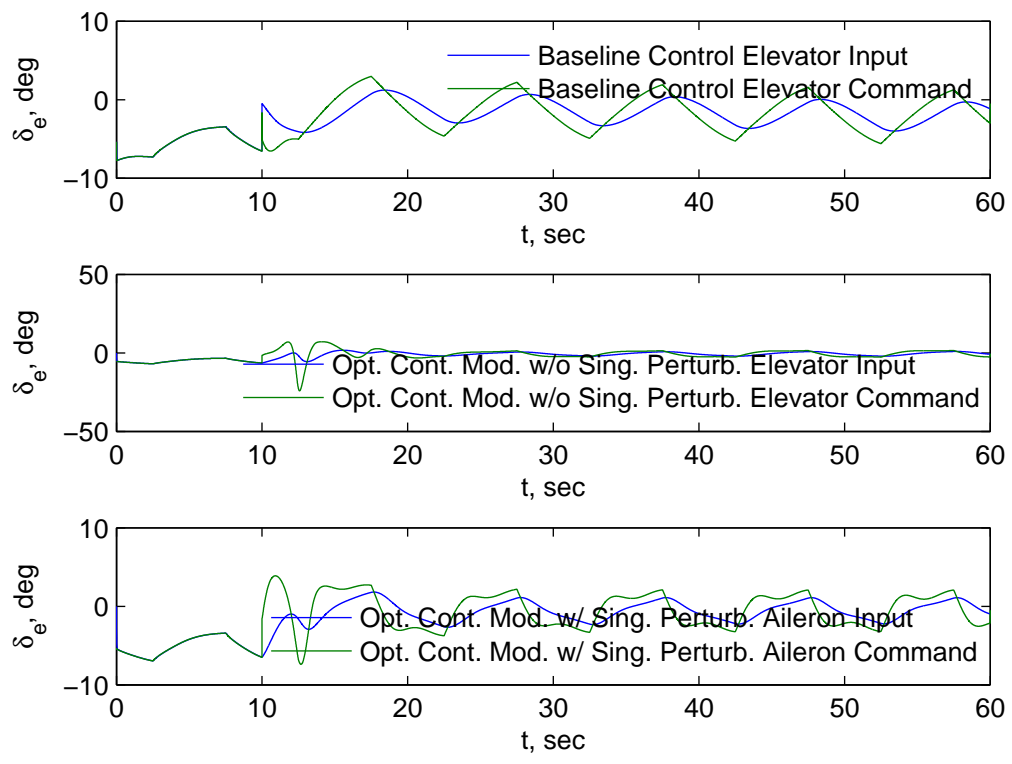

Fig. 11 - Elevator Deflection

The rudder deflection is shown in Fig. 12. With no adaptation, the rudder deflection oscillates from about $0^{\circ}$ to $-7^{\circ}$. Both the optimal control modification adaptive laws produce very similar rudder deflections, which reduce steadily from the initial transient to its new trim value of $-1^{\circ}$. 

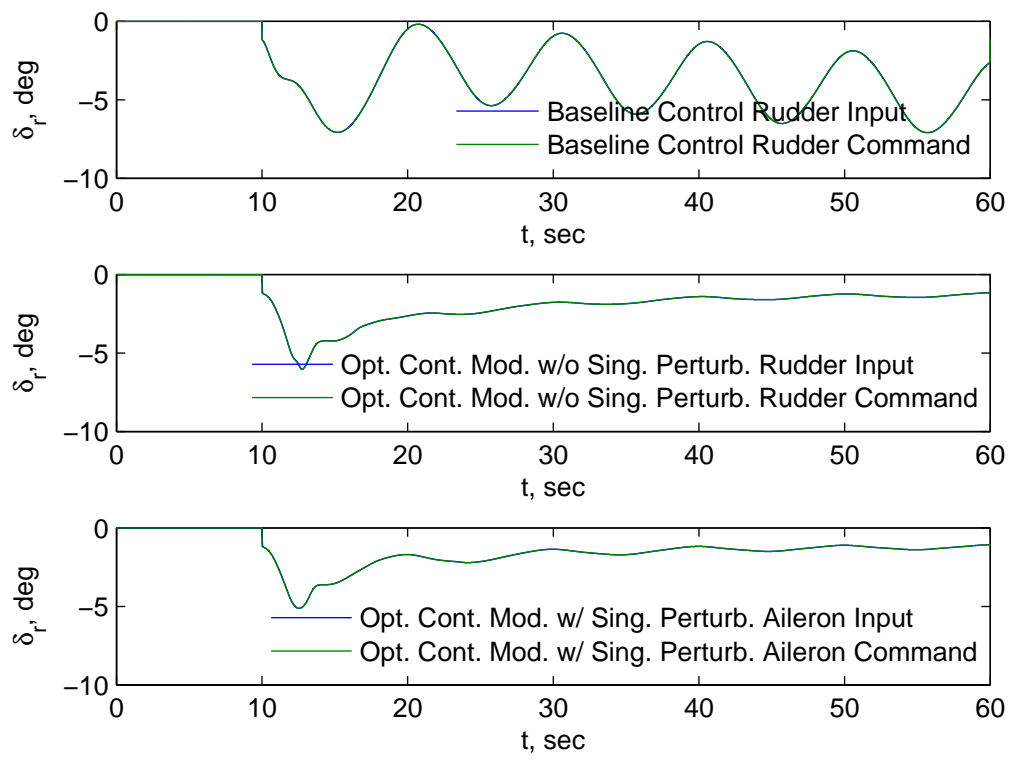

Fig. 12 - Rudder Deflection

The attitude responses of the damaged aircraft are shown in Figs. 13 to 16. When there is no adaptation, the pitch attitude could not be followed accurately as seen in Fig. 13. With the adaptation on, the tracking is much improved and the optimal control modification adaptive law performs better with than without the singular perturbation approach.
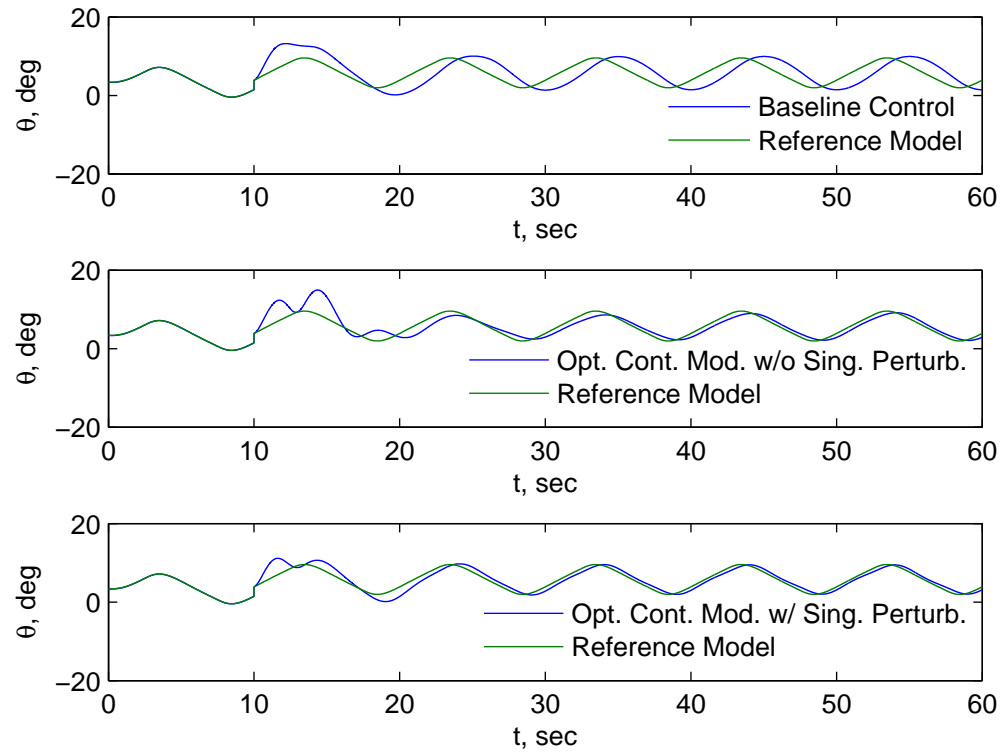

Fig. 13 - Pitch Angle

Figure 14 is the plot of the bank angle. Without the adaptation, the damaged aircraft exhibits a rather severe roll behavior with the bank angle ranging from $-40^{\circ}$ to $20^{\circ}$. With the adaptation on, the roll angle is drastically reduced from an initial transient of about $-30^{\circ}$ without and $-25^{\circ}$ with the singular perturbation approach to almost zero. 

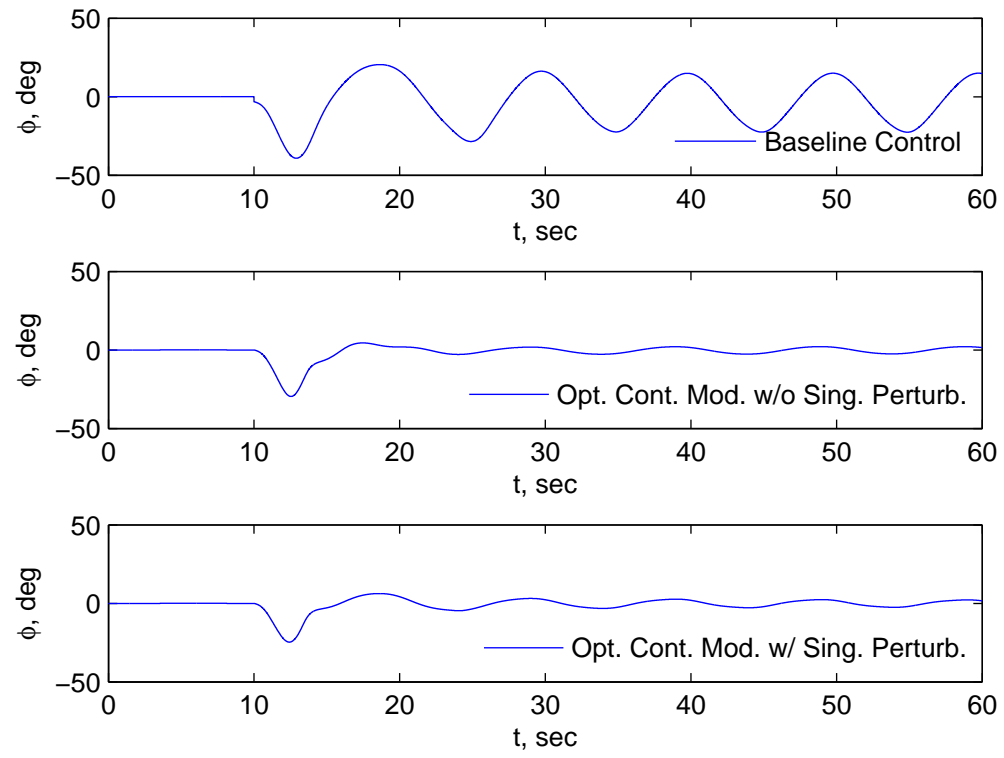

Fig. 14 - Bank Angle

Figure 15 is a plot of the angle of attack. The baseline controller results in a significantly large initial angle of attack when the damage occurs. The maximum angle of attack is about $12^{\circ}$, which could be close to stall. The maximum angle of attack is reduced to $6^{\circ}$ when the adaptation is on.
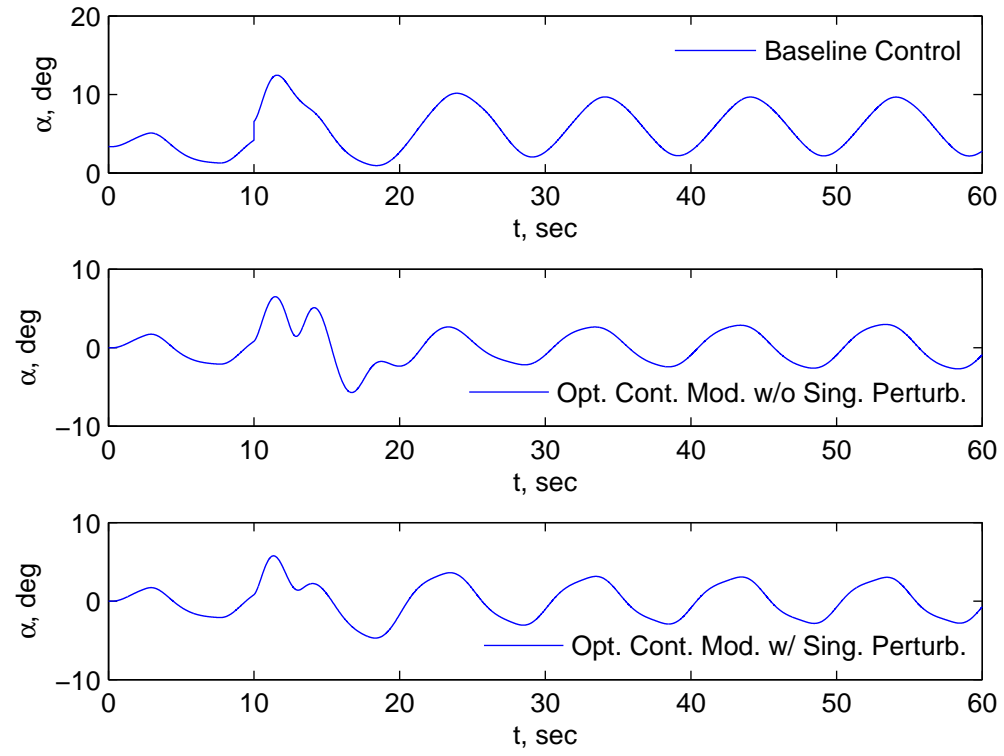

Fig. 15 - Angle of Attack

Figure 16 shows a plot of the sideslip angle.The baseline controller produces a steady oscillation in the sideslip angle between $\pm 2^{\circ}$. With both the optimal control modification adaptive laws, the sideslip angle is reduced to near zero. 

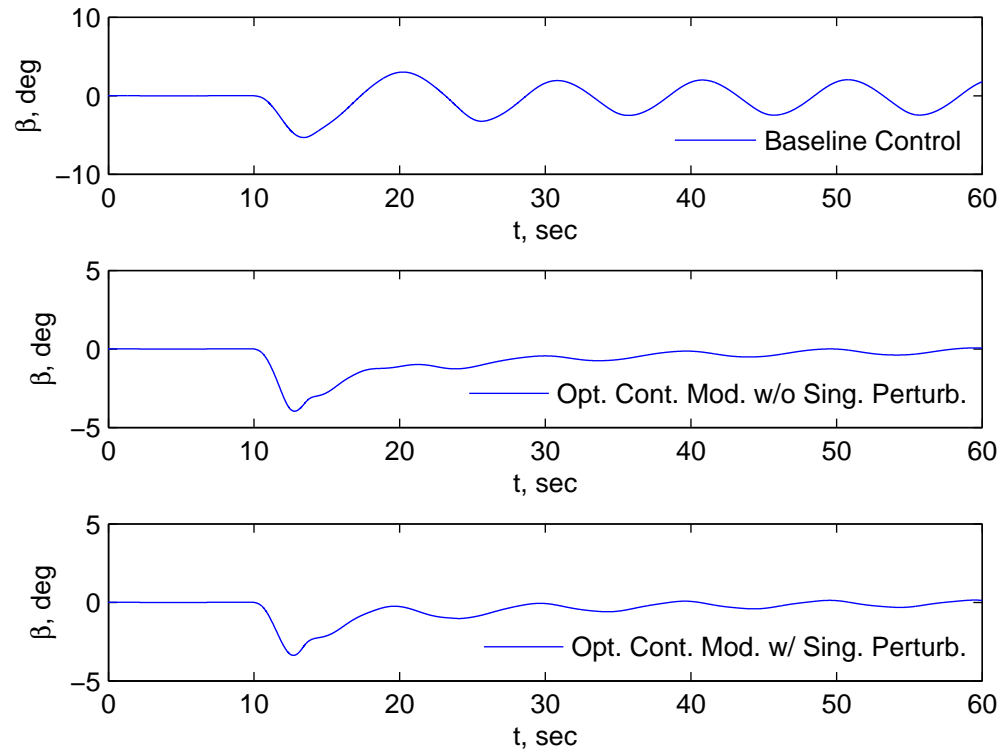

Fig. 16 - Sideslip Angle

\section{Conclusions}

This paper presents a singular perturbation approach in connection with an optimal control modification adaptive law for a time-scale separated system with slow actuator dynamics. The singular perturbation approach transforms the system into a reduced-order system in a slow time coordinate. The actuator command is obtained by the model matching condition in the slow time coordinate.The resulting actuator signal in effect is increased by the ratio of the norm of the plant's transition matrix to the norm of the slow actuator's transition matrix. The optimal control modification adaptive is derived and analyzed for stability using the Lyapunov method. Under fast adaptation when the adaptive gain is large, the analysis shows that the tracking error remains bounded and stable. The singular perturbation approach with the optimal control modification adaptive law is extended to a flight control application. Simulations of a flight control performance for a damaged aircraft with an impaired elevator actuator demonstrates the effectiveness of the method.

\section{References}

${ }^{1}$ Bosworth, J. and Williams-Hayes, P.S., "Flight Test Results from the NF-15B IFCS Project with Adaptation to a Simulated Stabilator Failure", AIAA Infotech@Aerospace Conference, AIAA-2007-2818, 2007.

${ }^{2}$ Johnson, E.N., Calise, A.J., El-Shirbiny, H.A., and Rysdyk, R.T., "Feedback Linearization with Neural Network Augmentation Applied to X-33 Attitude Control", AIAA Guidance, Navigation, and Control Conference, AIAA-2000-4157, 2000.

${ }^{3}$ Rohrs, C.E., Valavani, L., Athans, M., and Stein, G., "Robustness of Continuous-Time Adaptive Control Algorithms in the Presence of Unmodeled Dynamics", IEEE Transactions on Automatic Control, Vol AC-30, No. 9, pp. 881-889, 1985.

${ }^{4}$ Jenkins, D.R., "Hypersonics before the Shuttle: A Concise History of the X-15 Research Airplane", NASA Special Publication, SP-20004518, June 2000, Monographs in Aerospace History: No. 18.

${ }^{5}$ Eberhart, R.L. and Ward, D.G., "Indirect Adaptive Flight Control System Interactions", International Journal of Robust and Nonlinear Control, Vol. 9, pp. 1013-1031, 1999.

${ }^{6}$ Rysdyk, R.T. and Calise, A.J., "Fault Tolerant Flight Control via Adaptive Neural Network Augmentation", AIAA Guidance, Navigation, and Control Conference, AIAA-1998-4483, 1998.

${ }^{7}$ Cao, C. and Hovakimyan, N., "Design and Analysis of a Novel L1 Adaptive Control Architecture with Guaranteed Transient Performance", IEEE Transactions on Automatic Control, Vol. 53, No. 2, pp. 586-591, 2008.

${ }^{8}$ Nguyen, N., Krishnakumar, K., Kaneshige, J., and Nespeca, P., "Flight Dynamics and Hybrid Adaptive Control of Damaged Aircraft", AIAA Journal of Guidance, Control, and Dynamics, Vol. 31, No. 3, pp. 751-764, 2008.

${ }^{9}$ Nguyen, N. and Boskovic, J., "Bounded Linear Stability Margin Analysis of Nonlinear Hybrid Adaptive Control", Proceeding of 2008 IEEE American Control Conference, June 2008.

${ }^{10}$ Ioannu, P.A. and Sun, J. Robust Adaptive Control, Prentice-Hall, 1996. 
${ }^{11}$ Ioannou, P. and Kokotovic, P., "Instability Analysis and Improvement of Robustness of Adaptive Control," Automatica, Vol. 20, No. 5, 1984, pp. 583-594.

${ }^{12}$ Narendra, K.S. and Annaswamy, A.M., "A New Adaptive Law for Robust Adaptation Without Persistent Excitation”, IEEE Transactions on Automatic Control, Vol. AC-32, No. 2, pp. 134-145, 1987.

${ }^{13}$ Nguyen, N., Krishnakumar, K., and Boskovic, J., "An Optimal Control Modification to Model-Reference Adaptive Control for Fast Adaptation", AIAA Guidance, Navigation, and Control Conference, AIAA 2008-7283, 2008.

${ }^{14}$ Kokotovic, P., Khalil, H., and O'reilly, J., Singular Perturbation Methods in Control: Analysis and Design, Society for Industrial and Applied Mathematics, 1987.

${ }^{15}$ Ardema, M., "Computational Singular Perturbation Method for Dynamical Systems", AIAA Journal of Guidance, Control, and Dynamics, Vol. 14, 661-663, 1981. 1989.

${ }^{16}$ Cybenko, G., "Approximation by Superpositions of a Sigmoidal Function”, Mathematics of Control Signals Systems, Vol. 2, pp. 303-314,

${ }^{17}$ Bryson, A.E. and Ho, Y.C., Applied Optimal Control: Optimization, Estimation, and Control, John Wiley \& Sons Inc., 1979.

${ }^{18}$ Khalil, H.K., Nonlinear Systems, Prentice-Hall, 2002.

${ }^{19}$ Slotine, J.-J. E. and Li, W., Applied Nonlinear Control, Prentice-Hall, 1991.

${ }^{20}$ Narendra, K.S. and Annaswamy, A.M., Stable Adaptive Systems, Dover Publications, 2005.

${ }^{21}$ Shah, G., "Aerodynamic Effects and Modeling of Damage to Transport Aircraft", AIAA Atmospheric Flight Mechanics Conference, AIAA 2008-6203, 2008. 